Rev. Elev. Méd. vét. Pays trop., 1971, 24 (3) : 419-44

\title{
Essais d'embouche du Zébu Malgache
}

\author{
par H. SERRES $\left({ }^{*}\right)$, J. GILIBERT $(*)$, P. DUBOIS $\left(^{*}\right)$, \\ B. de REVIERS (*), J. TARDIF (*)
}

\begin{abstract}
RESUME
Des boufs Zébus Malgaches ont été soumis à une alimentation intensive basće sur l'utilisation du maïs, du tourteau d'arachide et des issues de riz.

Les animaux s'y adaptent très bien à l'exception des bêtes très âgées (plus de 10 ans). On peut obtenir des croîts quotidiens moyens approchant $700 \mathrm{~g}$ par jour pendant 4 mois, après quoi ils diminuent.

Les veaux au sevrage seraient les plus avantageux, mais ils ne sont pas traditionnellement commercialisés.

L'utilisation d'animaux âgés de 5 ans environ paraît la plus souhaitable. Elle permet d'obtenir d'excellentes carcasses atteignant facilement $180 \mathrm{~kg}$. Le poids de $200 \mathrm{~kg}$ est difficile à réaliser, compte tenu du format modeste des zébus malgaches.

La tuberculose risque de constituer un inconvénient sérieux.

Néanmoins, par un choix judicieux des animaux et des aliments l'opération peut être rentable.
\end{abstract}

\section{INTRODUCTION}

Madagascar possède dans ses régions NordOuest, Ouest et Sud d'immenses savanes. Elles se situent sous un climat typiquement tropical, avec alternance de saisons pluvieuses d'été et sèches d'hiver; leur végétation s'est développée sur des sols presque toujours acides, et les graminées qui sont largement dominantes ont une flambée de croissance en début des pluies puis ne tardent pas à mûrir pour donner la majeure partie de l'année une savane pailleuse.

La valeur alimentaire des pâturages de savanes de Madagascar, dominés par Hyparrhenia et Heteropogon contortus, est caractérisée par une haute teneur en cellulose, un faible pourcentage en matières azotées et en calcium. On peut dire qu'il s'agit de fourrages très grossiers, de valeur énergétique assez faible et qui, en dehors de la saison des pluies, entraînent une alimentation fortement carencée en azote.

(*) I.E.M.V.T., Région de recherches de Madagascar.
Le Zébu Malgache s'est séculairement adapté à ces conditions naturelles car l'homme n'a ici pratiquement rien fait pour aider son bétail (pas de gardiennage des troupeaux, de transhumance, ni de réserve).

Cette race s'est donc naturellement sélectionnée vers de faibles besoins; elle présente un format plutôt petit et léger aggravé par une remarquable tardivité, la taille adulte n'étant guère atteinte avant 7 ans. Par contre l'animal s'est adapté à l'accumulation de réserves pendant la période favorable, ce qui lui permet ensuite de franchir la saison de disette. Il a donc acquis une aptitude métabolique à l'accumulation de graisse dans sa bosse et sous la peau.

Le Zébu Malgache assure la majeure part de la fourniture de viande pour une autoconsommation rurale importante et une consommation urbaine qui s'accroît avec la démographie, l'urbanisation et le niveau de vie.

Le bétail né dans les savanes de l'Ouest est acheminé à pied vers les villes dont les principales sont, soit sur l'arête dorsale du Pays: 
Tananarive, Antsirabe, Fianarantsoa, soit sur la Côte Est: Tamatave, Diégo-Suarez.

Le bétail, déjà maigre au départ une partie de l'année, n'est pas amélioré par les longues marches. Dans les abattoirs on obtient des carcasses généralement insuffisantes, dont l'utilisation à l'exportation fut longtemps limitée à la conserverie.

Un besoin de carcasses de qualité est néanmoins ressenti depuis longtemps et il s'accentue. C'est pourquoi autour des villes et notamment de Tananarive, se pratique de façon traditionnelle l'engraissement des bœufs maigres selon la technique dite du "bœuf de fosse ". En plus, dans les régions pourvues de meilleurs pâturages, on pratique l'embouche à l'herbe de bovins achetés à 3 - 4 ans et revendus en bon état deux ans plus tard. Mais ces deux activités n'intéressent qu'une faible partie du bétail abattu; il convient de l'élargir si l'on veut accroître la quantité et la qualité de la viande produite. C'est pourquoi nous avons entrepris une série d'essais pour tenter de déterminer une technique économique d'embouche des zébus malgaches, car on peut disposer, suivant les régions, de quantités très importantes de manioc, de maïs, d'issues de riz, de tourteau d'arachide et sous peu sans doute de graines de coton.

Les premiers essais ont été pratiqués au Centre de Kianjasoa, situé à l'ouest de Tananarive dans la zone d'approvisionnement de cette ville, dans une région où la culture du maïs a pris une forte extension. Une deuxième série d'essais a été faite au Centre de Miadana, voisin de Majunga, où la construction d'un. abattoir industriel est en projet. Dans cette région, la grande plaine rizicole de Marovoay permet d'avoir des issues et des pailles de riz en grandes quantités.

Avant les expériences que nous allons décrire, une seule a été réalisée à Kianjasoa, il y a dix ans, et rapportée par METZGER et HAMON (1959). Six zébus pris au sevrage, alimentés de fourrages verts ou secs selon la saison, de manioc et d'une provende à $167 \mathrm{~g}$ de M.A.D. par U.F. ont grossi de $346 \mathrm{~g}$ par jour pendant 602 jours. Le crô̂t quotidien moyen maximal s'est élevé à $411 \mathrm{~g}$ pendant une période de 126 jours.

L'observation des bœufs de fosse, engraissés à l'herbe et au manioc, qui prennent environ
$100 \mathrm{~kg}$ en 200 jours (soit environ $500 \mathrm{~g}$ par jour) peut nous servir de point de repère, bien qu'il ne s'agisse pas exactement du type d'animal que nous recherchons.

Dans un premier essai, nous avons nourri des animaux ad libitum avec un concentré équilibré et le minimum de fourrage, pour avoir une idée approchée de la capacité maximale d'accroissement des zébus.

Ce chiffre obtenu, la mise au point d'un rationnement devient possible. Il est alors appliqué à des animaux d'âges divers.

Enfin un essai d'alimentation économique basé sur la consommation d'issues de riz complète le travail ci-après.

On a visé au départ l'obtention de carcasses d'un poids de $200 \mathrm{~kg}$, suffisamment grasses pour être classées en première qualité. Car il semble, bien que l'on n'en soit pas certain, que ce type de carcasses serait le plus facilement commercialisable.

\section{MATERIEL ET METHODES}

\section{LES ANIMAUX}

Les animaux utilisés au cours des essais suivants sont des Zébus Malgaches, achetés sur les marchés traditionnels du bétail. Ils n'ont fait l'objet d'aucun choix zootechnique spécial, en dehors d'une apparente bonne santé. Chaque lot comportait au départ dix animaux, formant un ensemble aussi homogène que possible, ce qui a conduit à prendre sur le marché des animaux moyens. $\mathrm{Ce}$ problème a chaque fois été compliqué parce que nous n'avons mis en expérience que des animaux à réaction tuberculinique négative afin d'éviter l'incidence d'un paramètre pathologique non mesurable au cours des essais. Les animaux qui durent être éliminés pour réaction positive furent nombreux.

Tous les animaux en expérience étaient castrés, soit par leur propriétaire, soit par nousmêmes après l'achat.

Comme les animaux sont d'origine inconnue, leur âge précis est incertain. Il a été estimé selon les caractères extérieurs, notamment les cornes et les dents. 


\section{ALIMENTATION}

Les régimes auxquels ont été soumis les animaux étaient dans tous les cas du type intensif avec un maximum de concentré équilibré et un minimum de verdure ou de verdure et de paille de riz pour assurer le lest digestif.

A Kianjasoa, le concentré étant basé sur le maîs broyé complété par du tourteau d'arachide et des minéraux, à Miadana, sur les issues de riz enrichies en azote par de l'urée, et en calcium par des coquillages marins broyés.

Concentré Kianjasoa:

- Maïs . . . . . . . . . . 80

- Tourteau . . . . . . . . . . . 16

— Os verts . . . . . . . . . 2
- Coquilles . . . . . . . . . . 1

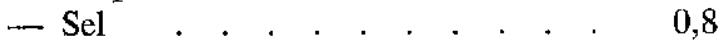

— Bicarbonate Na . . . . . . . 0,2

Concentré Miadana :

— Son de riz . . . . . . . . 32

- Farine basse de riz . . . . . 64

- Coquilles . . . . . . . . 1,5

... Urée . . . . . . . . . 1,5

- Sel . . . . . . . . . . . . 1

Les fourrages sont représentés par l'Herbe de Guatemala (Tripsacum laxum) et la variété Kisozi de l'Herbe à éléphant (Pennisetum purpureum). Ces deux espèces sont voisines comme type de fourrage et comme composition.

Les compositions chimiques des divers aliments utilisés sont rassemblées au tableau I.

TABLEAU $\mathbb{N}^{\circ} \mathrm{I}$

\begin{tabular}{|c|c|c|c|c|}
\hline Détermination & $\begin{array}{l}\text { Concentré } \\
\text { Kianjasoa }\end{array}$ & $\begin{array}{l}\text { Concentré } \\
\text { Miadana }\end{array}$ & Fourrage & $\begin{array}{l}\text { Paulle } \\
\text { de riz }\end{array}$ \\
\hline Eau & 8,6 & 8,7 & 81,3 & 9,9 \\
\hline Matières minërales & 4,4 & 11,3 & 1 & 15,2 \\
\hline Matières grasses & 4,6 & 11,4 & 0,4 & 1,4 \\
\hline $\begin{array}{c}\text { Matières azotées } \\
(\mathbb{N} \times 6,25)\end{array}$ & 14,9 & 14,3 & 1 & 4,5 \\
\hline Celluluse brute & 2,6 & 11,1 & 6,65 & $2 B, 3$ \\
\hline Extractif non azoté & 64,9 & 45,9 & 9,65 & 40,7 \\
\hline $\mathrm{Ca}$ & 0,9 & 0,9 & 0,04 & 0,3 \\
\hline $\mathrm{P}$ & 0,56 & 1,3 & 0,03 & 0,07 \\
\hline Valeur fourragère & 1,1 & 0,77 & 0,12 & 0,26 \\
\hline
\end{tabular}

Note

Le concentré de Miadana contient 1,5 p. 100 d'urée. Celle-ci intervient au dosage, selon Kjeldalh, pour un équivalent en matière azotée de 4,2 p. 100. La matière azotée non uréique de ce concentré est donc de 10,1 p. 100. Cela retentit sur le calcul de l'extractif non azoté, le chiffre à soustraire devenant alors $10,1+1,5$ $=11,6$. Le phosphore est abondant, mais apporté par les issues de riz il est en partie phytique, donc incomplètement utilisé.

\section{PROTOCOLES EXPERIMENTAUX}

1. Logement des animaux: A Kianjasoa comme à Miadana, les essais se sont déroulés en étable constituée d'un hangar métallique, fermé de tous côtés par une barrière tubulaire et divisé en trois stalles égales et contiguës de $10 \times 10 \mathrm{~m}$ chacune. Chaque animal disposait donc de $10 \mathrm{~m}^{2}$ de sol cimenté. Chaque stalle a $10 \mathrm{~m}$ de rateliers, $15 \mathrm{~m}$ de mangeoires et un abreuvoir important. Cela permet à tous les animaux d'avoir un large accès à la nourriture, et évite les bousculades. Les installations ne permettent pas un contrôle individuel de la nourriture. On soulignera que si les animaux sont à l'abri de la pluie et du soleil chaud, ils ne sont nullement protégés contre le vent.

2. Distribution de la nourriture: La nourriture est pesée journellement pour chaque lot tant en ce qui concerne le concentré que les fourrages. Le fourrage est distribué en une seule fois dans la matinée après qu'on l'ait coupé. On a remarqué que le fourrage tron- 
çonné en fragments de $30 \mathrm{~cm}$ au coupe-coupe est plus apprécié que le même fourrage passé au hache paille, en morceaux de $2 \mathrm{~cm}$. Le concentré est distribué en deux fois : le matin de bonne heure puis au début de l'après-midi. Les refus éventuels sont pesés tous les jours avant la distribution suivante.

Dans le cas d'alimentation ad libitum, on distribue une quantité de concentré telle qu'il ne soit jamais totalement utilisé.

La quantité de fourrage distribué ne dépasse jamais $8 \mathrm{~kg}$ par jour et par animal.

3. Pesées du bétail: Toutes les pesées se font à la bascule, le matin avant la distribution de nourriture. Les animaux sont pesés toutes les semaines, mais pour les poids utilisés dans les calculs on fait une pesée trois jours de suite et le poids au jour donné est la moyenne des trois (comprenant la veille et le lendemain). On limite ainsi, en grande partie, des erreurs provoquées par un abreuvement, une miction ou une défécation éventuelles, juste avant la pesée.

Les pesées de référence se font à intervalles assez longs pour niveler les irrégularités quotidiennes de la croissance. Dans la mesure du possible un intervalle de 4 semaines a été respecté.

4. Etude des carcasses: Les animaux engraissés à Kianjasoa ont été abattus à Tananarive, et ceux engraissés à Miadana ont été abattus à Majunga.

Les transports ont été effectués par camion. De nombreux relevés ont été faits sur les carcasses. Ils seront développés dans une étude ultérieure sur la qualité des carcasses de Zébus Malgaches.

En fonction de ces relevés on a indiqué les indices suivants :

- Rendement commercial : pourcentage du poids vif au départ représenté par la carcasse chaude.

- Rendement à jeun : pourcentage de poids vif avant abattage représenté par la carcasse chaude.

- Rendement vrai : pourcentage du poids vif vide représenté par la carcasse froide.

- Indice d'état de viande : c'est le rapport du poids de la carcasse froide en $\mathrm{kg}$, à la longueur de la carcasse en $\mathrm{cm}$. Il est d'autant plus élevé que l'animal est plus musclé et plus gras.

- Indice de muscle : c'est le rapport de l'épaisseur de la cuisse à la longueur de la carcasse. Il donne une appréciation de l'importance des masses musculaires de la carcasse car il est peu influencé par l'état d'engraissement.

- Indice de gras : c'est le rapport du poids du gras de rognons à celui de la carcasse froide. C'est le meilleur moyen d'apprécier l'état d'engraissement des animaux.

- Rendement en muscle : il n'a été effectué que pour quelques lots. C'est le pourcentage de carcasse représentée par les masses musculaires après désossage et parage du gras de façon industrielle.

5. Présentation des résultats: Les résultats sont groupés en tableaux qui sont divisés en deux parties. La première donne les gains de poids, les consommations et les indices de consommation par période, ce qui permet de suivre l'évolution du comportement des animaux.

La seconde partie est synthétique et cumule les gains comme les consommations depuis le début de l'essai. Elle conduit à des crôts quotidiens moyens cumulatifs et à des indices de consommation également cumulatifs qui intéressent l'économie de l'opération.

Les poids et gains individuels permettent d'apprécier les importantes variations individuelles qui sont d'ailleurs assez semblables d'un. lot à un autre.

\section{Note}

Il est à remarquer que les derniers poids des tableaux ne sont pas strictement égaux aux " poids au départ du Centre ". Ces derniers ont été pris au moment de la mise en camion. Or un délai a été imposé entre les derniers relevés et les abattages, par nécessité d'accord avec l'abattoir (essais Kianjasoa). Pendant ce temps les animaux ont pris quelques kilogrammes supplémentaires.

A Miadana, au contraire, les animaux ont été abattus dès la fin des expériences et embarqués après une nuit de jeûne; les "poids au départ du Centre » sont alors un peu plus bas. 


\section{RESULTATS EXPERIMENTAUX}

\section{ESSAIS REALISES A KIANJASOA}

\section{Essai préliminaire avec nourriture ad libitum}

Dix Zébus Malgaches de 3 à 4 ans environ ont été nourris ainsi avec le concentré maïstourteau défini au chapitre précédent. Le fourrage leur a été distribué à raison de $8 \mathrm{~kg}$ par jour. Les animaux se sont assez vite habitués à leurs changements de vie et nourriture; après un mois ils démarraient une croissance régulière.

Les résultats techniques sur la croissance sont rassemblés au tableau II (p. 426).

Plusieurs constatations s'imposent :

- D'abord un indice de consommation élevé, pratiquement toujours supérieur à 10 , lié au mode d'alimentation ad libitum.

- Des gains quotidiens moyens qui s'établissent à $593 \mathrm{~g}$ par jour pour une période de 6 mois environ.

On en déduit que la croissance du Zébu Malgache alimenté intensivement peut se situer autour de $600 \mathrm{~g}$ par jour pour une période de 6 mois environ. Par contre, elle peut atteindre $700 \mathrm{~g}$ par jour si elle est limitée à 4 mois.

Cette observation nous conduit à des normes de rationnement qui, cumulant la croissance et l'entretien, doivent se situer dans les fourchettes suivantes :

- de 200 à $250 \mathrm{~kg}, 5$ à 5,5 U.F./jour;

— de 250 à $300 \mathrm{~kg}, 5,5$ à $6 \quad$ U.F./jour;

— de 300 à $350 \mathrm{~kg}, 6$ à 6,5 U.F./jour.

Ce sont ces normes qui ont servi de base à la suite des essais.

- On note que les gains quotidiens moyens ne s'abaissent pas très régulièrement et on constate une légère dépression en juin. Cela correspond à un coup de froid survenu à ce moment-là. Au Centre de Kianjasoa, situé à 900 mètres d'altitude, il arrive que le vent d'alizé souffle fort les nuits d'hiver alors que la température est inférieure à $10^{\circ} \mathrm{C}$. Les zébus y paraissent sensibles. Ils restent longtemps couchés et mangent moins. Leur croissance s`en ressent.
- On constate que les gains journaliers en fin d'essai ont nettement diminué pour s'établir à $378 \mathrm{~g} /$ jour in fine.

Les animaux étaient alors très gras, et la transformation des aliments s'est faite avec un indice défayorable.

Sur le plan individuel les gains de poids ont été les suivants $(\mathrm{kg})$ :

$\begin{array}{llllllllll}386 & 328 & 372 & 399 & 328 & 351 & 330 & 388 & 340 & 400\end{array}$

$\begin{array}{llllllllll}241 & 246 & 266 & 269 & 223 & 262 & 220 & 242 & 232 & 258\end{array}$

$\begin{array}{llllllllll}145 & 82 & 106 & 130 & 105 & 89 & 110 & 146 & 108 & 142\end{array}$

Moyenne: $116 \mathrm{~kg}$.

Le gain le plus faible se situe à 56 p. $100 \mathrm{du}$ plus élevé.

Les animaux ont été abattus et les carcasses examinées à Tananarive ont donné les résultats suivants (moyenne par animal) :

TABLEAU $N^{\circ}$ III

\begin{tabular}{|ll|c|}
\hline Poids vif au dëpart & (kg) & 368 \\
Poids vif vide & $(\mathrm{kg})$ & 327 \\
Contenus digestifs & $(\mathrm{kg})$ & 41 \\
\multicolumn{1}{c|}{ p.100 de P.V. départ } & 11,1 \\
Poids carcasse chaude (kg) & 223 \\
Poids carcasse froide & $(\mathrm{kg})$ & 217,4 \\
Rendement commercial & $(\mathrm{p} .100)$ & 59 \\
Rendement à jeun & $(\mathrm{p} .100)$ & 63,5 \\
Rendement vrai & $(\mathrm{p} .100)$ & 66,4 \\
Longueur carcasse & $(\mathrm{cm})$ & 111,5 \\
Indice d'et at de viande & 1,95 \\
Epaisseur de la cuisse & $(\mathrm{cm})$ & 21,9 \\
Indice de muscle & $(\mathrm{p} .100)$ & 19,6 \\
Gras de rognons & $(\mathrm{kg})$ & 8,35 \\
Indice de gras & $(\mathrm{p} .100)$ & 3,8 \\
Rendement en muscle & $(\mathrm{p} .100)$ & 58,4 \\
\hline
\end{tabular}

Les carcasses des animaux étaient très grasses, la couverture très épaisse. Les bosses et les gras de rognons représentaient une proportion élevée de la carcasse. La viande a été très appréciée sur le marché de Tananarive où l'animal très gras est recherché. Toutefois, pour l'économie de l'opération d'embouche, il serait sans doute souhaitable d'obtenir des animaux un peu moins gras, coûtant moins cher en aliments. 
TABLEAU $\mathrm{N}^{\circ} \mathrm{II}$

Engraibsement au maís-tourteau d'arachide ad libitzm (10 animaux de 3 à 4 ans)

\begin{tabular}{|c|c|c|c|c|c|c|c|c|c|c|c|c|c|c|c|}
\hline$D$ a $\mathbf{t}$ e $\mathbf{s}$ & 1.03 & & 30.3. & & 26.4 . & & 24.5 . & & 21.6. & & 19.7. & & 16.8 & & 13.9. \\
\hline Poids du 1ot & 2459 & & 2647 & & 2917 & & 3090 & & 3234 & & 3394 & & 3516 & & 3622 \\
\hline Gaingpar période $(\mathrm{kg})$ & & 188 & & 270 & & 173 & & 144 & & 160 & & 122 & & 106 & \\
\hline C.Q.M. par période(g.) & & 671 & & 964 & & 617 & & 514 & & 571 & & 435 & & 378 & \\
\hline U.F. par pêriode & & 2180 & & 2524 & & 2552 & & 2115 & & 2239 & & 2108 & & 1746 & \\
\hline I.C. par période & & 11,6 & & 9,34 & & 14,75 & & 14,68 & & 14 & & 17,2 & & 16,47 & \\
\hline Gains cumulés & & & 188 & & 458 & & 631 & & 775 & & 935 & & 1057 & & 1163 \\
\hline C.Q.M. cumulatifs (g.) & & & 671 & & 817 & & 751 & & 691 & & 667 & & 629 & & 593 \\
\hline U.F. cumulêes & & & 2180 & & 4704 & & 7256 & & 9371 & & 11610 & & 13718 & & 15464 \\
\hline I.C. cumulatifs & & & 11,6 & & 10,6 & & 11,5 & & 12,1 & & 12,4 & & 12,97 & & 13,3 \\
\hline
\end{tabular}

TABLEAU $\mathrm{N}^{\circ} \mathrm{IV}$

Engraissement au maîs-tourteau rationné (10 animaux 2 ans $1 / 2$ )

\begin{tabular}{|c|c|c|c|c|c|c|c|c|c|c|c|c|c|c|c|}
\hline$D$ a $t$ e $s$ & 7.02 & & 20.4 & & 12.6 & & 21.8 & & 16.10 & & 11.12 & & 9.01 . & & 24.02 . \\
\hline Poids du lot & 1730 & & 2278 & & 2541 & & 2780 & & 3002 & & 3304 & & 3394 & & 3529 \\
\hline Gains par période (kg) & & 548 & & 263 & & 239 & & 222 & & 302 & & 90 & & 135 & \\
\hline G.Q.M. pat période (g) & & 751 & & 496 & & 341 & & 396 & & 539 & & 321 & & 293 & \\
\hline U.F. par période & & 3926 & & 3553 & & 4376 & & 3651 & & 3861 & & 1897 & & 3005 & \\
\hline I.C. par përiode & & 7,2 & & 13,5 & & 18,3 & & 16,4 & & 12,8 & & 21,1 & & 22,3 & \\
\hline Gains cumrlés & & & 548 & & 811 & & 1050 & & 1272 & & 1574 & & 1664 & & 1779 \\
\hline C.Q.M. cumulatifs $(\mathrm{kg})$ & & & 751 & & 644 & & 536 & & 505 & & 511 & & 495 & & 471 \\
\hline U.F. cumulëcs & & & 3926 & & 7479 & & 11855 & & 15506 & & 19367 & & 21264 & & 24269 \\
\hline I.C. cumulatifs & & & 7,2 & & 9,2 & & 11,3 & & 12,2 & & 12,3 & & 12,8 & & 13,5 \\
\hline
\end{tabular}




\section{Essais avec rationnement}

\section{A. Animaux jeunes}

Il est bien connu que les animaux ont un croît quotidien plus élevé et un indice de consommation plus faible s'ils sont plus jeunes. Nous avons donc testé les possibilités des plus jeunes bêtes que l'on peut trouver sur les marchés : âgées de deux ans à deux ans et demi. Au-dessous de cet âge là, les jeunes qui subissent durement la contrainte du milieu dans l'année qui suit le sevrage, ne sont pas déplacés vers les marchés. Le rationnement comportait $4 \mathrm{~kg}$ de concentré par jour avant $200 \mathrm{~kg}$, $5 \mathrm{~kg}$ après $200 \mathrm{~kg}$ et $5,5 \mathrm{~kg}$ après $300 \mathrm{~kg}$. Les croîts quotidiens ayant diminué, la ration n'a pas été augmentée au franchissement des $250 \mathrm{~kg}$.

L'examen des résultats techniques rassemblés au tableau IV montre que les animaux ont démarré une croissance rapide pendant trois mois $(751 \mathrm{~g} /$ jour); puis la croissance a connu des irrégularités. Certaines phases de faibles gains quotidiens (juin-juillet $=252 \mathrm{~g} /$ jour) peuvent trouver une explication partielle dans le rôle du froid auquel les zébus paraissent sensibles. Toutefois des essais complémentaires (animaux en plein air, animaux en étable close et alimentés de la même façon) nous ont montré qu'il ne convenait pas de surestimer ce facteur.

De toute façon, passé le quatrième mois, les croîts quotidiens se sont maintenus au-dessous de $500 \mathrm{~g} /$ jour, exception faite d'un clocher momentané (octobre).

Les indices de consommation se sont montrés, au début, très favorables pendant trois mois (aux alentours de 7). Puis ils se sont rapidement élevés, de sorte que l'indice cumulatif a franchi nettement le seuil de $10 \mathrm{au}$ sixième mois.

Il devenait certain que la rentabilité ne pouvait plus être assurée; l'essai a été cependant poursuivi pour se rapprocher de l'objectif fixé (carcasses de $200 \mathrm{~kg}$ ).

Les gains de poids individuels sont relativement homogènes.

$$
\begin{array}{rllllllllll}
\text { Poids en fin d'essai . . } & 366 & 338 & 332 & 369 & 391 & 365 & 378 & 327 & 361 & 302 \\
\text { Poids au début d'essai : } & 185 & 176 & 160 & 170 & 180 & 188 & 160 & 172 & 179 & 160 \\
\cline { 2 - 9 } \text { Gains : } & 181 & 162 & 172 & 199 & 211 & 177 & 218 & 155 & 182 & 142 \\
\multicolumn{8}{c}{\text { Moyenne : } 179,9 .}
\end{array}
$$

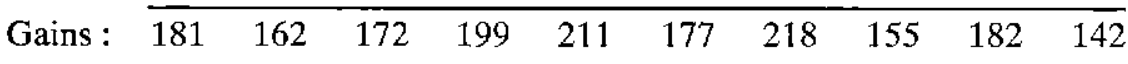

\begin{tabular}{|ll|c|}
\hline \multicolumn{3}{|c|}{ TABLEAU $\mathrm{N}^{\circ} \mathrm{VI}$} \\
\hline Poids vif au départ & (kg) & 356,3 \\
Poids vif vide & $(\mathrm{kg})$ & 324,2 \\
Contenus digestifs & $(\mathrm{kg})$ & 32,1 \\
p.100 p.v. départ & 9 \\
Poids carcasse chaude & $(\mathrm{kg})$ & 209,5 \\
Poids carcasse froide & $(\mathrm{kg})$ & 206,7 \\
Rendement commercial & $(\mathrm{p} .100)$ & 58,8 \\
Rendement à jeun & $(\mathrm{p} .100)$ & 61,5 \\
Rendement vrai & $(\mathrm{p} .100)$ & 63,7 \\
Longueur carcasse & $(\mathrm{cm})$ & 110,8 \\
Indice d'état de viande & 1,86 \\
Epaisseur de la cuisse & $(\mathrm{cm})$ & 22,6 \\
Indice de muscle & $(\mathrm{p} .100)$ & 20,4 \\
Gras de rognons & $(\mathrm{kg})$ & 8,9 \\
Indice de gras & $(\mathrm{p} .100)$ & 4,3 \\
Rendement en muscle & $(\mathrm{p} .100)$ & 57,2 \\
\hline
\end{tabular}

Le gain le plus faible est de 65 p. 100 du plus fort.

Les carcasses ont donné les résultats notés au tableau VI.

Les animaux étaient très couverts, les bosses et les gras de rognons étaient excédentaires. Le poids de carcasse recherché a été atteint, et même légèrement dépassé à cause du rendement en carcasses particulièrement élevé.

\section{B. Animaux d'âge moyen (3 à 4 ans environ)}

10 zébus de 3 à 4 ans environ d'un poids moyen voisin de $230 \mathrm{~kg}$ ont été mis en engraissement. Le rationnement a comporté $5 \mathrm{~kg}$ de concentré avant $250 \mathrm{~kg}$ et $6 \mathrm{~kg}$ ensuite.

Les bouvillons se sont bien adaptés à leur sort sauf l'un d'entre eux qui refusait de man- 


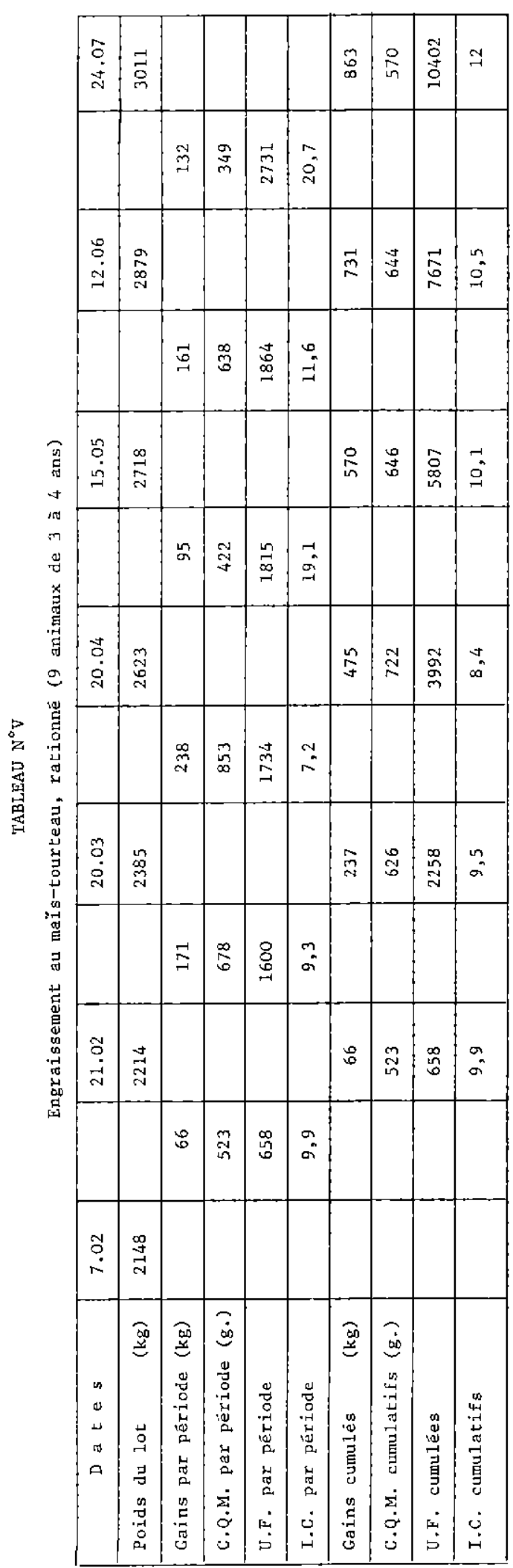


ger, perdait du poids et dut être éliminé. Cette éventualité d'animaux ne s'adaptant pas est à noter.

Les résultats de la croissance et des consommations des animaux sont consignés au tableau $\mathrm{n}^{\mathrm{o}} \mathrm{V}$.

On constate que les gains sont élevés pendant les trois premiers mois. Puis on retrouve comme pour le lot précédent une diminution où joue peut-être le froid. Le parallélisme des phénomènes observés dans les deux lots nous porte à les imputer à la météorologie.

Les animaux du lot que nous considérons avaient le même âge, ont été nourris dans les mêmes conditions et à la même saison que le lot ad libitum de l'année précédente. Une comparaison est donc permise. On constate que dans l'ensemble les gains de poids sont peu différents. Par contre, par suite du rationnement les indices de consommation sont nettement plus favorables.

Sur le plan individuel on relève les gains de poids suivants :

$\begin{array}{lllllllll}338 & 380 & 317 & 321 & 275 & 351 & 366 & 351 & 312\end{array}$ $\begin{array}{lllllllll}258 & 260 & 217 & 250 & 200 & 239 & 254 & 250 & 220\end{array}$

$\begin{array}{lllllllll}80 & 120 & 100 & 71 & 75 & 112 & 112 & 101 & 92\end{array}$

Moyenne : 86,3.

Le gain le plus faible est à 59 p. 100 du plus élevé.

Les animaux ont été abattus à Tananarive et ont donné en moyenne par animal:

\section{TABLEAU $\mathrm{N}^{*} \mathrm{VII}$}

\begin{tabular}{|ll|c|}
\hline Poids vif au départ & $(\mathrm{kg})$ & 341 \\
Poids vif vide & $(\mathrm{kg})$ & 286,8 \\
Contenus digestifs & $(\mathrm{kg})$ & 54,2 \\
\multicolumn{1}{|c}{ p.loo P.v. départ } & & 15,9 \\
Poids carcasse chaude & $(\mathrm{kg})$ & 180,6 \\
Poids carcasse froide & $(\mathrm{kg})$ & 179,2 \\
Rendement contuercial & $(\mathrm{p} .100)$ & 52,9 \\
Rendement à jeun & $(\mathrm{p} .100)$ & 57,5 \\
Rendement vrai & $(\mathrm{p} .100)$ & 62,5 \\
Longueur de la carcasse & $(\mathrm{cm})$ & 111 \\
Indice d'état de viande & & 1,61 \\
Epaisseur de la cuisse & $(\mathrm{cm})$ & 21,1 \\
Indice de muscle & $(\mathrm{p} .100)$ & 19 \\
Gras de rognons & $(\mathrm{kg})$ & 3,88 \\
Indice de gras & $(\mathrm{p} .100)$ & 2,2 \\
Rendement en muscle & $(\mathrm{p} .100)$ & 59 \\
\hline
\end{tabular}

Comme on le voit, le rendement était moyen. La couverture était incomplète, les bosses pas très développées, le gras de rognon également. Les animaux ont été abattus parce que leurs derniers gains quotidiens venaient de passer en dessous de $400 \mathrm{~g}$.

Cet essai n'a pas permis d'obtenir des carcasses de $200 \mathrm{~kg}$ de moyenne.

\section{Animaux âgés}

Un lot d'animaux âgés de 7 à 10 ans a été utilisé. Ils avaient tous atteint leur taille maximale et ils étaient, à la mise à l'étable, en état relativement bon. Aucun n'était vraiment très maigre. L'adaptation a été un peu plus difficile qu'avec les animaux plus jeunes, car centaines intolérances individuelles ont persisté plusieurs semaines durant. Mais aucun incident majeur n'en a résulté et l'essai a pu se dérouler normalement pendant 4 mois.

Le rationnement a comporté $5 \mathrm{~kg}$ de concentré avant $350 \mathrm{~kg}, 6 \mathrm{~kg}$ entre 350 et $400 \mathrm{~kg}$, $6,5 \mathrm{~kg}$ après $400 \mathrm{~kg}$.

Les résultats en sont retracés au tableau VIII. On remarque une croissance plus importante au cours du deuxième mois qu'au cours du premier. Cela est à imputer à une prolongation de l'adaptation chez ces bêtes âgées. Puis les performances diminuent pour demeurer très intéressantes. Après quatre mois le crô̂t quotidien moyen de $660 \mathrm{~g} /$ jour est parmi les plus élevés que nous ayons obtenus.

Par contre l'indice de consommation est toujours voisin de 10 . Il atteint 13.8 le dernier mois et 10,7 pour l'ensemble des 4 mois.

Deux animaux ont été vendus sur le marché de bétail de manière à connaître les prix qu'ils peuvent obtenir.

Ils ont trouvé preneur â 50 FMG le $\mathrm{kg}$.

Malheureusement ils n'ont pu être ensuite contrôlés au point de vue carcasses et les chiffres qui suivent se rapportent aux 8 autres bœufs du lot. (tabl. IX).

Bien que les poids des bosses et des gras de rognons paraissent assez faibles, les animaux étaient correctement engraissés. Ils avaient une couverture, quoique légère, régulièrement répartie. Tous, sauf un, sont passés en première qualité. Le seul qui ait été classé en $2^{e}$ qualité était le plus léger et il se trouve 
TABLEAU N ${ }^{a}$ VIII

Engraissement au maĩs-tourteau, rationné (10 animaux âgés de 7 à 10 ans)

\begin{tabular}{|c|c|c|c|c|c|c|c|c|c|}
\hline $\mathrm{D}$ a $\mathrm{te} \mathrm{s}$ & 14.01 & & 12.02 & & 12.03 & & 9.04 & & 7.05 \\
\hline Poids du lot $\quad(\mathrm{kg})$ & 3310 & & 3460 & & 3700 & & 3886 & & 4050 \\
\hline Gains par përiode $(\mathrm{kg})$ & & 150 & & 240 & & 186 & & 164 & \\
\hline C.O.M. par përiode $(\mathrm{g})$ & & 535 & & 857 & & 664 & & 585 & \\
\hline U.F. par përiode & & 1621 & & 2000 & & 2045 & & 2262 & \\
\hline I.C. par période & & 10,8 & & 8,33 & & 11 & & 13,8 & \\
\hline Gains cumulës $\quad(\mathrm{kg})$ & & & 150 & & 390 & & 576 & & 740 \\
\hline C.Q.M. cumulatifs (g) & & & 535 & & 696 & & 685 & & 660 \\
\hline U.F. cumulées & & & 1621 & . & 3621 & & 5666 & & 7928 \\
\hline I.C. cumulatifs & & & 10,8 & & 9,3 & & 9,8 & & 10,7 \\
\hline
\end{tabular}

TABLEAU N ${ }^{\circ}$ IX

\begin{tabular}{|llc|}
\hline Poids vif au départ & $(\mathrm{kg})$ & 410,6 \\
Poids vaf vide & $(\mathrm{kg})$ & 348,5 \\
Contenus digestifs & $(\mathrm{kg})$ & 62,1 \\
\multicolumn{1}{c}{ p.100 P.V. dêpart } & & 15,1 \\
Poids carcasse chaude & $(\mathrm{kg})$ & 220 \\
Poids carcasse froide & $(\mathrm{kg})$ & 216,3 \\
Rendement conmercial & $(\mathrm{p} .100)$ & 53,6 \\
Rendement à jeun & $(\mathrm{p} .100)$ & 56,3 \\
Rendement vrai & $(\mathrm{p} .100)$ & 62 \\
Longueur carcasse & $(\mathrm{cm})$ & 122,4 \\
Indice d'état de viande & & 1,77 \\
Epaisseur de la cuisse & $(\mathrm{cm})$ & 22,9 \\
Indice de muscle & $(\mathrm{p} .100)$ & 18,7 \\
Gras de rognons & $(\mathrm{kg})$ & 3,28 \\
Indice de gras & $(\mathrm{p} .100)$ & 1,48 \\
\hline
\end{tabular}

qu'il était assez fortement parasité par Fasciola gigantica.

On soulignera aussi que dans la liste ci-après, les animaux $n^{\circ} 3$ et $n^{\circ} 7$ étaient porteurs de caséifications tuberculeuses pulmonaires peu étendues. L'un d'eux a eu le gain de poids le plus facile, l'autre un gain nettement inférieur à la moyenne.

Les gains de poids individuels se répartissent comme suit :

$$
\mathrm{n}^{\circ} 3 \quad \mathrm{n}^{0} 7
$$

$\begin{array}{llllllllll}432 & 402 & 418 & 401 & 419 & 432 & 370 & 359 & 418 & 399\end{array}$ $\begin{array}{llllllllll}357 & 321 & 355 & 340 & 341 & 332 & 316 & 298 & 331 & 319\end{array}$

$\begin{array}{llllllllll}75 & 81 & 63 & 61 & 78 & 100 & 54 & 61 & 87 & 80\end{array}$ (douve)
Moyenne: $74 \mathrm{~kg}$.

Le gain de poids le plus faible est à 54 p. 100 du plus élevé.

\section{ESSAIS CONDUITS A MIADANA}

Le Centre de Miadana est situé au voisinage de Majunga, à une altitude voisine du niveau de la mer; le climat est vraiment tropical.

1. Animaux d'âge moyen (5 ans environ)

L'engraissement de deux lots de 10 animaux de cinq ans environ y a été pratiqué à l'aide de l'alimentation à base d'issues de riz qui a été décrite précédemment.

L'un des lots a été soumis à un régime dit " haut ", l'autre à un régime dit "bas".

Dans tous les cas les animaux recevaient journellement $7 \mathrm{~kg}$ de fourrage vert et $2 \mathrm{~kg}$ de paille de riz par tête. Les quantités du mélange concentré distribuées étaient les suivantes :

TABLEAU $N^{\circ} \mathrm{X}$

\begin{tabular}{|c|c|c|}
\hline & Avant $300 \mathrm{~kg}$ & Après $300 \mathrm{~kg}$ \\
\hline "haut" & $5,2 \mathrm{~kg}$ & $6,2 \mathrm{~kg}$ \\
"bas" & $4,2 \mathrm{~kg}$ & $5,2 \mathrm{~kg}$ \\
\hline
\end{tabular}

Pour donner une idée plus précise des animaux, nous donnons ci-après les mensurations moyennes pour chaque lot: 
TABLEAU $\mathrm{N}^{\circ} \mathrm{XI}$

\begin{tabular}{|c|c|c|c|}
\hline & $\begin{array}{c}\text { Longueur } \\
\text { Scapulo ischiaie } \\
\text { (cm) }\end{array}$ & $\begin{array}{c}\text { Hauteur au } \\
\text { garrot (cm) }\end{array}$ & $\begin{array}{c}\text { Hauteur au } \\
\text { sacrum (cm) }\end{array}$ \\
\hline Lot "Bas" & 139 & 115 & 124 \\
Lot "Haut" & 138 & 116 & 124 \\
\hline
\end{tabular}

Les bœufs se sont adaptés normalement à la claustration en 3 semaines environ.

Comme les deux lots sont aussi près que possible l'un de l'autre au départ, nous étudierons synthétiquement leur évolution.

Le mélange à base d'issues de riz, utilisé mouillé au moment de la distribution, a été bien mangé par les animaux. Le lot "Bas " n'a pratiquement jamais laissé de refus, alors que pour le lot "Haut", on a noté par périodes quelques refus qui ont été déduits. Ils ont surtout été observés dans les jours qui ont suivi l'augmentation de la ration, après le franchissement des $300 \mathrm{~kg}$.

- On constate que pour les deux lots les évolutions des différents paramètres se sont faites de façon régulière.

A Miadana, la température est toujours chaude et les animaux n'ont subi le préjudice d'aucune intempérie.

- Les croîts globaux ont été équivalents pour les deux lots sur l'ensemble de l'essai. Cela confirme que pour un engraissement prolongé, un niveau d'alimentation "Haut "n'est pas rentable.

- Les croîts quotidiens moyens ont été d'abord plus élevés avec le lot au niveau " Haut", mais, après trois mois, la situation s'est inversée. Le niveau "Haut" serait concevable pour un engraissement court de 3 mois, même s'il entraîne un indice de consommation un peu plus élevé car ce dennier serait compensé (au moins en partie) par le gain de temps.

- A l'issu de l'essai les animaux étaient très gras, et il aurait fallu arrêter l'engraissement un mois plus tôt.

Néanmoins on a éprouvé des difficultés, comme nous allons le voir, à atteindre les $200 \mathrm{~kg}$ de carcasses recherchés.
- Les indices de consommation sont, au moins pour le niveau bas, meilleurs que pour le lot d'animaux de 4 ans engraissé à Kianjasoa. Le climat y est sans doute pour partie.

Sur le plan des augmentations individuelles de poids on obtient en $\mathrm{kg}$ :

«Bas »:

$\begin{array}{llllllllll}337 & 288 & 344 & 372 & 384 & 367 & 354 & 350 & 303 & 335\end{array}$

$\begin{array}{lllllllllll}240 & 250 & 289 & 267 & 312 & 281 & 260 & 281 & 243 & 248\end{array}$

$\begin{array}{llllllllll}92 & 38 & 55 & 105 & 72 & 86 & 94 & 69 & 60 & 87\end{array}$

(tub.)

Moyenne : $75,8 \mathrm{~kg}$.

« Haut »:

$\begin{array}{llllllllll}294 & 390 & 344 & 380 & 332 & 353 & 302 & 340 & 358 & 339\end{array}$

$\begin{array}{llllllllll}260 & 315 & 248 & 277 & 240 & 261 & 250 & 268 & 275 & 270\end{array}$

$\begin{array}{llllllllll}34 & 75 & 96 & 103 & 92 & 92 & 52 & 72 & 83 & 69\end{array}$

(tub.)

Moyenne: $76,8 \mathrm{~kg}$.

C'est ici que nous devons ouvrir une parenthèse importante: malgré l'épreuve tuberculinique négative, un animal anergique a développé une tuberculose clinique en fin d'essai (toux, essoufflement, etc.). Il se trouve que cet animal est celui qui a pris le moins de poids : $34 \mathrm{~kg}$ du lot "Haut ». Dès que l'animal a été dépisté cliniquement une retuberculination de tous les animaux a été faite : 13 ont ćté trouvés fortement réagissants par suite de la contagion.

A l'abattage, on a recherché les lésions avec soin. L'animal cliniquement atteint en fin d'essai a subi une saisie totale pour tuberculose miliaire aiguë généralisée. Un deuxième animal présentait des lésions pulmonaires évolutives qui ont fait saisir la fressure. Il se trouve que cet animal a eu la croissance la plus faible du lot "Bas" (38 kg). Treize animaux ont présenté des lésions ganglionnaires très localisées, apparemment non évolutives, insuffisantes pour avoir diminué l'état général et Ia croissance. 
TABLEAU $N^{\circ} \mathrm{XII}$

Engraissement au son de riz - Niveau bas

\begin{tabular}{|c|c|c|c|c|c|c|c|c|c|c|c|c|c|}
\hline D a $t$ e $s$ & 1.06 & & 22.06 & & 20.07 & & 17.08 & & 14.09 & & 12.10 & & 9.11 . \\
\hline Poids du lot $\quad(\mathrm{kg})$ & 2676 & & 2764 & & 2991 & & 3138 & & 3272 & & 3400 & & 3434 \\
\hline Gains par pêriode(kg) & & 88 & & 227 & & 147 & & 134 & & 128 & & 34 & \\
\hline C.Q.M. par période (g) & & 419 & & 810 & & 525 & & 478 & & 457 & & 121 & \\
\hline U.F. par përiode & & 987 & & 1316 & & 1430 & & 1544 & & 1535 & & 1539 & \\
\hline I.C. par période & & 11,2 & & 5,8 & & 9,7 & & 11,5 & & 12 & & 45 & \\
\hline Gains cumulës $\quad(\mathrm{kg})$ & & & 88 & & 315 & & 462 & & 596 & & 724 & & 758 \\
\hline C.Q.M. cumulatifs (g) & & & 419 & & 646 & & 600 & & 567 & & 544 & & 471 \\
\hline U.F. cumulées & & & 987 & & 2303 & & 3733 & & 5277 & & $68] 2$ & & 8351 \\
\hline I.C. cunulatifs & & & 11,2 & & 7,3 & & 8,1 & & 8,8 & & 9,4 & & 11 \\
\hline
\end{tabular}

TABLEAU $\mathrm{N}^{\circ} \mathrm{XI}$ II

Engraissement au son de riz - Niveau haut

(10 animaux de 5 ans)

\begin{tabular}{|c|c|c|c|c|c|c|c|c|c|c|c|c|c|}
\hline D a $t$ e $s$ & 2.06 & & 22.6 & & 20.7 & & 17.8. & & 14.9 & & 12.10 & & 9.11. \\
\hline Poids du lot (kg) & 2664 & & 2803 & & 3011 & & 3168 & & 3289 & & 3405 & & 3432 \\
\hline Gains pax période $(\mathrm{kg})$ & & 139 & & 208 & & 157 & & 121 & & 116 & & 27 & \\
\hline C.Q.M. par période (g) & & 661 & & 742 & & 560 & & 432 & & 414 & & 96 & \\
\hline U.F. par pếriode & & 1155 & & 1540 & & 1604 & & 1755 & & 1808 & & 1722 & \\
\hline I.C. par période & & 8,3 & & 7,4 & & 10,2 & & 14,5 & & 15,6 & & 63,7 & \\
\hline Gains cumulês $\quad(\mathrm{kg})$ & & & 139 & & 347 & & 504 & & 625 & & 746 & & 768 \\
\hline C.Q.M. cumulatifs (g) & & & 661 & & 708 & & 654 & & 595 & & 557 & & 477 \\
\hline U.F. cumulêes & & & 1155 & & 2695 & & 4299 & & 6054 & & 7862 & & 9584 \\
\hline I.C. cumulatifs & & & 8,3 & & 7,7 & & 8,5 & & 9,7 & & 10,6 & & 12,5 \\
\hline
\end{tabular}


Les résultats d'abattages ont donné les résul- tats suivants en moyenne par animal:

TABLEAU $\mathrm{N}^{\circ} \mathrm{XIV}$

\begin{tabular}{|c|c|c|c|}
\hline & & Lot "Bas" & Lot "Haut" \\
\hline Poids vie au départ & $(\mathrm{kg})$ & 337 & 341 \\
\hline Poids carcasse chaude & $(\mathrm{kg})$ & 191,4 & 198,1 \\
\hline Poids cartasse froude & $(\mathrm{kg})$ & 187,6 & 194,8 \\
\hline Rendement conmercial & $(p \cdot 100)$ & 56,8 & 58,1 \\
\hline Longueur de la carcasse & $(\mathrm{cm})$ & 115,8 & 113 \\
\hline Indice d'état de viande & & 1,62 & 1,72 \\
\hline Eparsseur de la cursse & $(\mathrm{cm})$ & 22 & 23,3 \\
\hline Indice de muscle & $(p \cdot 100)$ & 18,9 & 20,6 \\
\hline Gras de rognons & $(\mathrm{kg})$ & 3,4 & 3,6 \\
\hline Indice de gras & $(p \cdot 100)$ & 1,77 & 1,82 \\
\hline
\end{tabular}

Le rendement en carcasses a été excellent Ies animaux étaient bien couverts et l'état d'engraissement très satisfaisant. On remarque un rendement un peu plus élevé, ainsi qu'un peu plus de gras pour le lot alimenté au niveau " Haut ".

A noter que les moyennes pour le lot "Haut" sont établies sur 9 carcasses, celle qui a été saisie pour tuberculose n'ayant pas fait l'objet de mesures. Les moyennes du lot en sont donc quelque peu avantagées. Bien que contrarié par la tuberculose, cet essai est cependant d'un haut intérêt pratique, car un tel phénomène se reproduira obligatoirement dans un centre d'embouche. C'est pourquoi nous donnons néanmoins le détail des résultats.

\section{Animaux très âgés (de 10 à 15 ans)}

Cet essai a intéressé deux lots de 10 boufs grands et vieux, achetés en très mauvais état, vraiment très maigres.

Les deux lots ont disposé toujours du même concentré à base d'issues de riz. Le lot "A" ne disposait pas de fourrage vert. Celui-ci était remplacé par $3 \mathrm{~kg}$ de paille de riz chaque jour. quantité que l'on a dû ramener à $2 \mathrm{~kg}$ car elle limitait la consommation de concentré.

Afin d'éviter une carence vitaminique chaque bœuf a reçu au cours du premier mois une injection apportant:

- Vitamine A

1.000 .000 U. I.

- Vitamine $\mathrm{D}_{3}$

1.000 .000 U. I.

- Vitamine B
- Vitamine $\mathrm{K}$

$30 \mathrm{mg}$

Le lot " $\mathbf{B}$ " recevait comme dans les essais précédents $8 \mathrm{~kg}$ par jour de fourrage vert. Le concentré a été distribué à raison de $5,2 \mathrm{~kg}$ par jour avant $350 \mathrm{~kg}$ et $6,2 \mathrm{~kg}$ après $350 \mathrm{~kg}$.

Nous soulignerons le comportement médiocre des animaux. L'adaptation à la stabulation permanente a été mauvaise. Dans le lot " $\mathbf{B}$ " deux animaux refusèrent de manger et ils ont dû être abattus in extremis avant que ne débute l'essai. C'est pourquoi à la date du 9 janvier le groupe « $B$ » ne comporte que 8 animaux.

Ensuite dans le lot " $\mathrm{A}$ ", un bœuf se montrait très agressif; il parvenait même à éventrer un congénère qui devait être retiré le 6 février. La décision de l'écorner fut alors prise. Mais à la suite de l'amputation pratiquée près du crâne, il développa une sorte de complexe d'infériorité et refusa de manger. Il dut être également éliminé.

Au 6 mars, deux animaux de chaque lot devaient encore être éliminés.

Somme toute le déroulement de ces expériences a été troublé par la très mauvaise adaptation des animaux. L'étude des gains de poids et des consommations est néanmoins retracée au tableau $\mathrm{XV}$.

Les chiffres cumulatifs ne sont pas présentés, car la disparition de plusieurs animaux en cours d'expérience leur retire leur sens. 
TABLEAU $\mathbb{N}^{\circ} \mathrm{XV}$

1 - Lot A (Zébus sans verdurc)

\begin{tabular}{|c|c|c|c|c|c|c|c|}
\hline Dates & 9.01 & & 6.02 & & 6.03 & & 3.04 \\
\hline Poids du lot $\quad(\mathrm{kg})$ & $3132(10)$ & & $\begin{array}{l}3463(10) \\
2838(8)\end{array}$ & & $\begin{array}{l}2959(8) \\
2418(6)\end{array}$ & & $2472(6)$ \\
\hline Gains par periode (kg) & & $\overline{331}$ & & 121 & & 54 & \\
\hline C.Q.M. par période & & 1180 & & 560 & & 316 & \\
\hline U.F. par pêriode & & 1263,60 & & 1271,20 & & 971,88 & \\
\hline I.C. par période & & 3,81 & & 10,50 & & 17,99 & \\
\hline 2 - Lot B (Zébus avec verdurc) & & & & & & & \\
\hline D a t e s & 9.01 & & 6.02 & & 6.03 & & 3.04 \\
\hline Poids du lot $\quad(\mathrm{kg})$ & $2493 \quad(8)$ & & $2745(8)$ & & $\begin{array}{l}2877(8) \\
2198(6)\end{array}$ & & $2294(6)$ \\
\hline Gains par période (kg) & & 252 & & 132 & & 96 & \\
\hline C.Q.M. par përiode & & 1125 & & 587 & & 566 & \\
\hline U.F. par période & & 984,8 & & 1337,56 & & 1042,44 & \\
\hline I.C. par période & & 3,9 & & 10,13 & & 10,86 & \\
\hline
\end{tabular}


Nous indiquerons encore ci-après les résultats d'abattoir donnés par quatre bœufs de chaque lot.

Les bœufs ont donné des rendements faibles, car les viscères abdominaux étaient très déve- loppés. La couverture était insuffisante.

Il faut conclure que l'engraissement d'animaux très âgés n'est pas à entreprendre en groupes. Les paysans qui pratiquent « en fosses " les traitent individuellement.

TABLEAU $N^{\circ} \mathrm{XVI}$

\begin{tabular}{|c|c|c|c|}
\hline & & Lot A & Lot $B$ \\
\hline Poids vif au depart & $(\mathrm{kg})$ & 402,5 & 398 \\
\hline Poids de carcasse chaude & $(\mathrm{kg})$ & 199,7 & 197,6 \\
\hline Poids de carcasse froide & $(\mathrm{kg})$ & 195,9 & 193,3 \\
\hline Rendement commercial & $(\mathrm{p} .100)$ & 49,3 & 49,6 \\
\hline Longueur de carcasse & (cm) & 126,5 & 124,5 \\
\hline Indice d'êtat de viande & & 1,55 & 1,55 \\
\hline Epaisseur de 1a cuisse & $(\mathrm{cm})$ & 22 & 22,4 \\
\hline Indice de muscle & $(p \cdot 100)$ & 17,4 & 18 \\
\hline Gras de rognons & $(\mathrm{kg})$ & 1,8 & 1,4 \\
\hline Indice de gras & $(p .100)$ & 0,9 & 0,7 \\
\hline
\end{tabular}

On remarque un indice de consommation très bas le premier mois. Cela est simplement dû à ce que les bœufs étaient très maigres, qu'ils se sont réhydratés et ont rempli leur panse, d'où un gain de poids considérable qui n'intéresse pas les masses musculaires. Puis les indices de consommation se sont maintenus au-dessus de 10.

Par ailleurs, pour un engraissement de courte durée il semble que la paille de riz suffise comme aliment de lest, sous réserve d'injecter à l'animal des vitamines $\mathbf{A}$ et $\mathbf{D}$.

\section{Abreuvement :}

La consommation d'eau a été mesurée pour les deux lots.

- Pour le lot A qui ne disposait que de paille comme aliment de lest, elle s'est élevée à 21 litres par jour et par animal.

- Pour le lot B qui consommait de la verdure, elle a été de 16 litres par jour et par animal.

\section{Engraissement de veaux}

Nous avons enfin testé la capacité de développement de veaux Zébus Malgaches, pris après le sevrage. Nous avons bénéficié d'un lot de plusieurs dizaines d’animaux, âgés en moyenne de dix mois et séparés récemment de leurs mères. Nous en avons choisi dix qui, par leur développement, se situaient dans la moyenne du lot. Les plus beaux et les plus faibles ont donc été écartés.

Ils ont été nourris, compte tenu de leur jeune âge, non avec le "Concentré Miadana" mais avec le "Concentré Kianjasoa" dans lequel, pour des raisons d'approvisionnement, le maïs a été remplacé par de la farine basse de riz. Le mélange minéral a été modifié en conséquence, car la farine basse est riche en phosphore. Nous rappelons que ce mélange ne fait pas appel à l'urée mais à du tourteau d'arachides. Sa valeur fourragère est voisine de 1 U.F. par $\mathrm{kg}$. Sa consommation a été limitée à $5 \mathrm{~kg}$ par jour.

Chaque jour il a été distribué $5 \mathrm{~kg}$ de verdure par tête, sous forme de Pueraria phaseolides, dont les grosses tiges délaissées étaient pesées.

On a constaté une adaptation très facile des animaux à la claustration et à leur régime alimentaire. Dès le début de l'essai les crôtts quotidiens ont été élevés et, durant trois mois environ, ils se sont maintenus proches de $1 \mathrm{~kg}$ par jour. Puis ils se sont abaissés d'abord à $500 \mathrm{~g}$ puis à $350 \mathrm{~g} /$ jour. A ce moment les animaux marchaient un peu "sur des épingles » 
TABLEAU N ${ }^{\circ}$ XVII

Engraissement de veaux zêbus Miadana 1969 - 1970 (10 animaux)

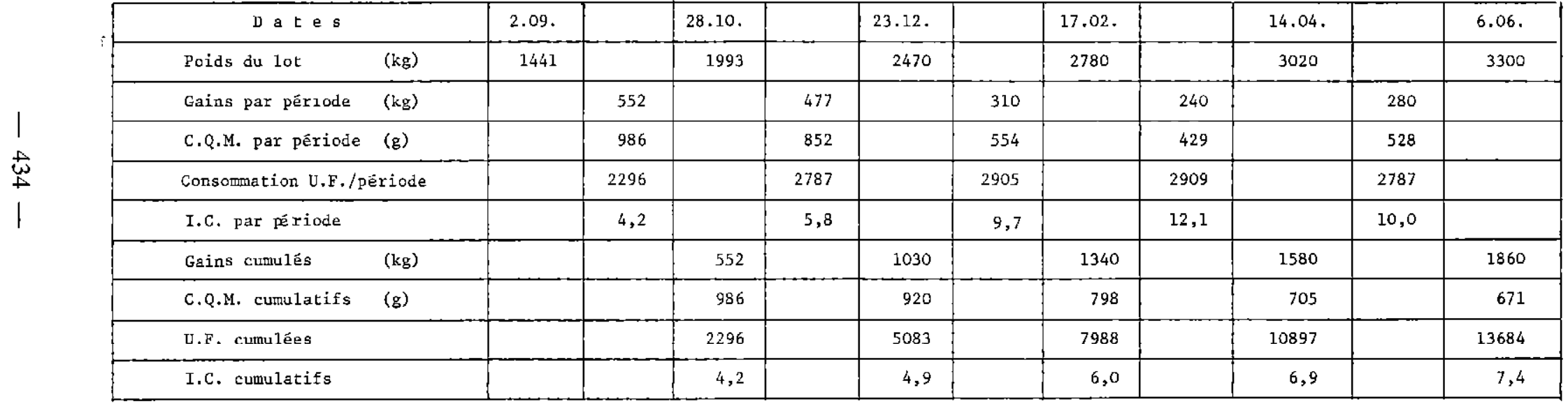


et présentaient un poil terne. Nous leur avons administré $1 \mathrm{~g}$ de sulfate de cuivre par jour, bien que l'analyse en oligo-éléments de la ration n'ait pas montré de déficit caractérisé en cuivre. Les croîts quotidiens sont alors remontés à $750 \mathrm{~g} /$ jour. Il est possible que l'administration de sulfate de cuivre en soit la cause mais ce n'est pas certain car un essai ultérieur n'a pas confirmé l'action positive de ce sel.

Par contre, il est apparu aux observateurs que la vitesse de croissance squelettique a été le facteur limitant. En effet, à l'issue des trois premiers mois les animaux étaient très arrondis, mais ils n'avaient pas beaucoup grandi et, surtout, par rapport à des animaux ayant du sang taurin, ne s'étaient pas élargis. Ils ne pouvaient, dès lors, maintenir un rythme élevé de croissance. Les résultats sont rassemblés au tableau XVII. Nous devons souligner la qualité des indices de consommation particulièrement bas. On a l'impression qu'au départ le comportement digestif des animaux a été celui de monogastriques, pour évoluer peu à peu vers celui de ruminant. Mais, malgré la diminution de la vitesse de croissance, l'indice général de consommation sur l'ensemble de l'essai est inférieur à 7,5. Cela contribue à la rentabilité de l'opération.

Les croissances individuelles ont donné les résultats suivants $(\mathrm{kg})$ :

\begin{tabular}{lllllllllll}
355 & 315 & 323 & 364 & 328 & 319 & 344 & 333 & 303 & 316 & \\
157 & 136 & 148 & 156 & 155 & 139 & 145 & 138 & 136 & 131 \\
\hline 198 & 179 & 175 & 208 & 173 & 180 & 199 & 195 & 167 & 185
\end{tabular}$\quad(\mathrm{~m}=186)$

La plus faible des croissances représente 80 p. 100 de la plus forte, ce qui traduit une homogénéité ni obtenue, ni approchée, avec aucun autre lot d'animaux plus âgés.

Pour des raisons indépendantes de notre volonté, l'abattage n'a pas pu être fait à Majunga. Un transport en camion à Tananarive $(550 \mathrm{~km})$ puis quatre jours d'attente sur place ont entraîné une importante perte de poids, consécutive à la vidange partielle des réservoirs digestifs, mais aussi à un amaigrissement certain. Les carcasses qui ont pesé en moyenne $174,2 \mathrm{~kg}$ auraient sans doute approché $180 \mathrm{~kg}$ dans des conditions normales, et le rendement 54,5 p. 100 .

On peut donc considérer que cet cssai de 9 mois nous a procuré des carcasses comparables à celles du lot "Animaux d'âge moyen 3 à 4 ans environ " traité à Kianjasoa pendant 5 mois et demi (tableau XVIII).

Les carcasses étaient bien couvertes. sans excès, avec une bosse un peu volumineuse et des gras de rognons assez légers. Elles ont été jugées excellentes. Sans doute la viande devaitelle être particulièrement tendre, les animaux n'ayant que 19 mois en moyenne.
TABLEAU $N^{\circ} \mathrm{XVIII}$

\begin{tabular}{|ll|c|}
\hline Ponds vif au départ & $(\mathrm{kg})$ & 330 \\
Poids vif vide & $(\mathrm{kg})$ & 280,8 \\
Contenus digestifs & $(\mathrm{kg})$ & 49 \\
p.100 P.V. départ & & 14,8 \\
Poids carcasse chaude & $(\mathrm{kg})$ & 174,2 \\
Poids carcasse froide & $(\mathrm{kg})$ & 171,9 \\
Rendement commercial & $(\mathrm{p} .100)$ & 52,8 \\
Rendement à jeun & $(\mathrm{p} .100)$ & 58,3 \\
Rendement vrai & $(\mathrm{p} .100)$ & 61,2 \\
Longueur de carcasse & $(\mathrm{cm})$ & 108,2 \\
lndice d'état de viande & & 1,58 \\
Epaisseur de la cusse & $(\mathrm{cm})$ & 21,1 \\
Indice de tnuscle & $(\mathrm{p} .100)$ & 19,5 \\
Gras de rognons & $(\mathrm{kg})$ & 2,56 \\
Indice de gras & $(\mathrm{p} .100)$ & 1,48 \\
Rendement en muscle & $(\mathrm{p} .100)$ & 58 \\
& & \\
\hline
\end{tabular}




\section{DISCUSSION}

\section{COMPORTEMENT GENERAL DU ZEBU MALGACHE}

Adaptation à la claustration: Bien que provenant d'un élevage très libre, les Zébus Malgaches se sont adaptés à la claustration d'une manière satisfaisante. Les délais de cette adaptation paraissent plus courts pour les animaux jeunes que pour les animaux âgés (20 jours au lieu de 30). Les animaux se calment et l'on peut assez vite les caresser. Cependant ils conservent toujours un comportement plus agité que les autres races, pures ou métisses, que nous avons pu observer dans des conditions semblables.

Adaptation à la nourriture concentrée : Sur ce plan le succès est total avec les animaux jeunes ou d'âge moyen. Au cours de nos essais aucun incident n'a été constaté. Le comportement digestif a toujours été excellent. On notera que certains animaux âgés de plus de 10 ans s'y habituent mal, mangent insuffisamment et n'engraissent pas.

Adaptation au climat: Les essais montrent nettement l'adaptation du zébu malgache au climat chaud; la sensibilité au froid hivernal des plateaux peut se traduire par des diminutions de la vitesse d'engraissement pendant les coups de froid.

Influence de l'homogénéité des lots: Il est important de ne mettre à l'embouche que des lots homogènes quant à la taille des animaux. Les seuls incidents que nous ayons rencontrés ont eu l'hétérogénéité pour cause. Les petits sont battus par les grands et peuvent dépérir.

Avec une homogénéité approximative et des mangeoires aux larges dimensions, les échauffourées des premiers jours se calment rapidement.

Pathologie: La tuberculose paraît pouvoir être un obstacle non négligeable, particulièrement à redouter si l'on envisage un engraissement de type long. La cohabitation favorise le contage, et sa prolongation peut entraîner la formation de lésions étendues. Un examen clinique soigneux devrait permettre de dépister les anergiques à l'épreuve tuberculinique.

Dans les régions où le parasitisme par la douve géante peut être rencontré, il sera néces- saire de traiter les bœufs contre ce parasite avant de les mettre à l'engraissement.

\section{CROISSANCE ET CONSOMMATION}

Pour juger des croissances obtenues dans les essais décrits ci-dessus, nous pensons nécessaire de les situer dans le cadre de la croissance normale du Zébu Malgache.

Dans les conditions naturelles, on peut considérer que le bœuf Zébu Malgache soumis à la disette saisonnière gagne au maximum $45 \mathrm{~kg}$ par an entre 1 an et 8 ans, ce qui donne un croît quotidien moyen d'environ 120 g par jour.

Lors d'essais non publiés, nous avons entretenu pendant 8 mois des zébus de 3 ans avec un excellent fourrage à volonté (Chloris gayana) quelques $\mathrm{kg}$ de légumineuses en vert (Stylosanthes gracilis) et $1 \mathrm{~kg}$ par jour de concentré : urée, poudre d'os, manioc. Les animaux se sont développés pendant l'essai, ont été en permanence en bon état, mais ils n'ont jamais montré de signe d'engraissement excessif. Leur croît quotidien moyen a été de $230 \mathrm{~g}$ par jour. On peut en conclure qu'un zébu malgache nourri convenablement peut s'accroître sans engraisser outre mesure, d'une façon nettement supérieure à oe que l'on observe dans la nature.

Lorsqu'on achète, comme nous l'avons fait, des bovins sur les marchés, on trouve donc des animaux qui tous sont en retard. Il s'ensuit que, mis en engraissement et l'adaptation étant faite, on obtient une croissance accélérée. Volontairement nous ne disons pas compensatrice, parce qu'elle est loin de compenser le retard accumulé. Pendant deux mois on peut obtenir des gains pouvant aller jusqu'à $800 \mathrm{~g}$ par jour et $1.000 \mathrm{~g}$ pour les veaux. Les indices de consommation sont pendant cette période très intéressants : voisins de 5 à 1 an, entre 7 et 8 pour des animaux de 2 ans, entre 8 et 9 pour coux de 4 à 5 ans, entre 9 et 10 pour les adultes.

Mais cette période ne dure pas, et après trois mois l'animal, quel que soit son âge, voit ses crôts quotidiens diminuer.

Le Zébu Malgache est un animal particulièrement tardif, et la vitesse de croissance de son squelette est lente, même si l'alimentation est satisfaisante; les facteurs génétiques y jouent un rôle prépondérant. 
Cela est d'autant plus net que les animaux sont mis en engraissement à un stade plus avancé de leur croissance. Mais même pour les veaux le phénomène s'est manifesté de façon très nette.

L'animal se met à engraisser, les croîts quotidiens diminuent et les indices de consommations augmentent.

C'est là que le jugement devient important: si l'on veut de bonnes carcasses, il faut s'engager dans la seconde période pour obtenir un degré d'engraissement souhaitable, mais il faut s'arrêter assez tôt pour ne pas compromettre l'économie de l'opération.

Il semble qu'il faille suivre de près l'engra'ssement pour éviter que les indices de consommation ne franchissent le seuil de 10 et les croîts quotidiens ne descendent au-dessous de $550 \mathrm{~g}$ par jour pour l'ensemble de l'engraissement.

Au plan des performances individuelies des ánimaux et en fonction de nos moyens expérimentaux, nous ne pouvons juger que les gains de poids.

Si dans chaque essai nous cotons 100 la moyenne des gains du lot, nous pouvons rapporter en pour cent de la moyenne les gains de chaque animal. Pour l'ensemble de cinq des lots abattus on obtient la distribution en classes suivantes :

On voit que les trois quarts environ des animaux sont compris entre $m-15$ p. 100 et $\mathrm{m}+30 \mathrm{p} \cdot 100$. La moyenne est, par rapport à ce groupe, dissymétriquement situéc vers la gauche et la distribution n'apparaît pas normale. Cela est dû à ce qu'un centain nombre d'animaux ont des croissances vraiment déficientes pour des raisons de pathologie inapparente. (Il serait intéressant de pouvoir les dépister précocement pour les éliminer au plus tôt.)

Pour l'instant on ne peut pas donner de critère assez sûr, et une étude complémentaire sur un nombre élevé d'animaux pourra peutêtre dégager certaines corrélations significatives.

\section{QUALITE DES CARCASSES}

Nous avons rassemblé au tableau XIX les caractéristiques principales des carcasses des différents lots étudiés. Le rapprochement nous permet d'effectuer quelques constatations.

1. La taille des carcasses s'accroit avec l'âge, ce qui est normal, mais cela se poursuit jusqu'à un âge très avancé qui est de l'ordre de 10 ans. C'est ce qui explique le choix d'animaux très âgés par les paysans malgaches qui désirent obtenir des carcasses très lourdes (bceuf de fosse).

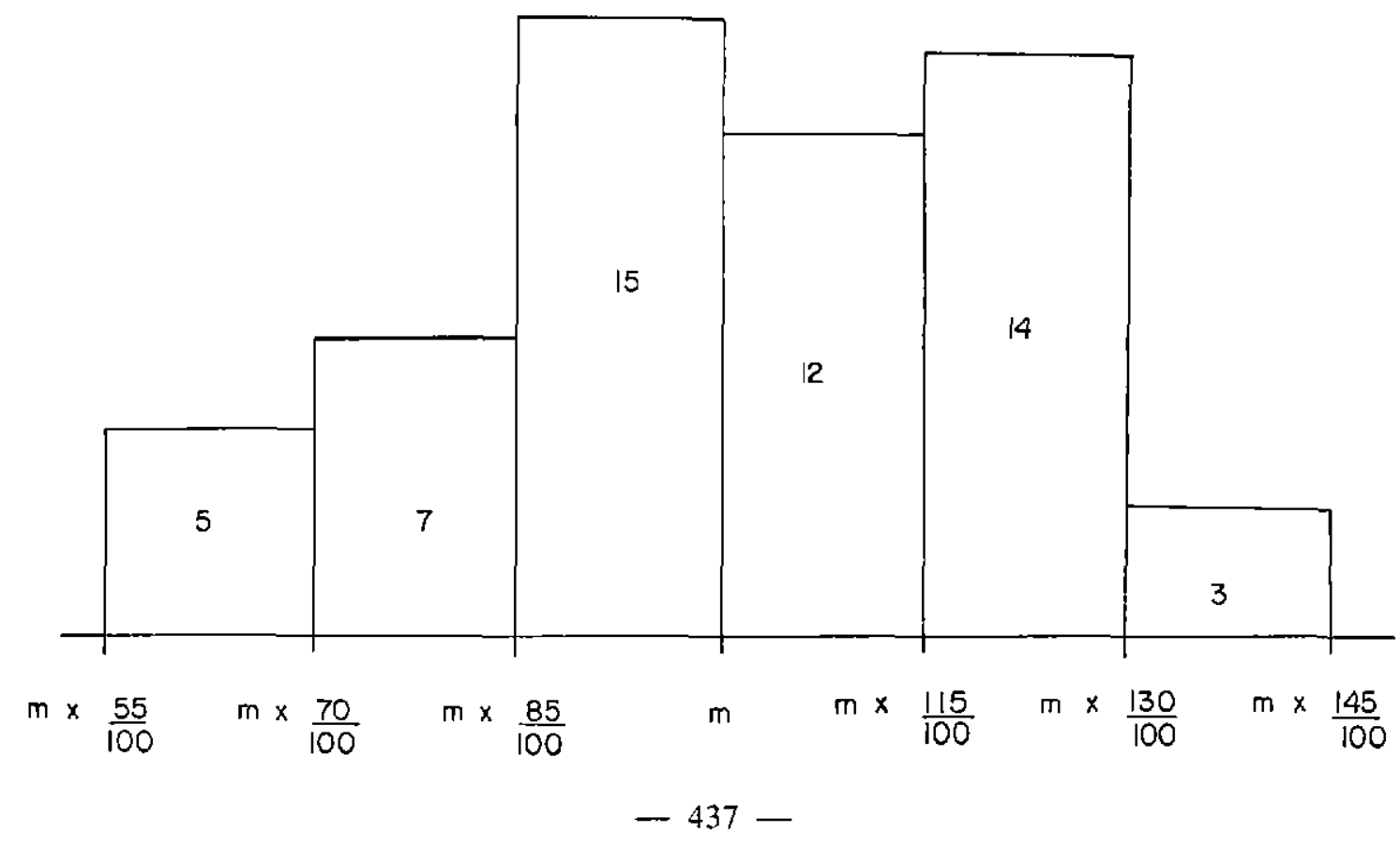


2. A degré d'engraissement égal, le poids des carcasses augmente avec leur taille et donc avec l'âge. Moyennant un engraissement non excessif, il est nécessaire d'utiliser des boufs ayant au moins six ans, si l'on veut obtenir avec un coût convenable des carcasses finies d'un poids de $200 \mathrm{~kg}$.

Ce poids de carcasse peut être obtenu avec des animaux plus jeunes ( 4 ans) mais au prix d'une durée d'embouche nettement prolongée et d'un état d'engraissement excessif; cela n'est pas à rechercher à cause du prix de revient et d'un excès de suif sur les carcasses destinées à l'exportation.

3. Le rendement commercial ne paraît guère influencé par l'âge des animaux, sauf en ce qui concerne ceux très âgés qui demandent à être fortement engraissés pour atteindre des rendements convenables ou élevés.

On remarque qu'au moyen d'un engraisse- ment non exagéré on obtient des rendements variant entre 53 et 56 p. 100 . Il se confirme donc qu'un rendement voisin de 55 p. 100 représente un optimum dont il convient de se rapprocher.

4. L'indice de muscle varie assez peu, mais il paraît, à engraissement égal, être un peu plus favorable chez les animaux jeunes (ce qui est normal, les tissus musculaires se développant plus tôt que les tissus adipeux). Dans la mesure du possible, il y aura donc intérêt à utiliser des animaux pas trop âgés; la tendreté de la viande en sera également bénéficiaire.

5. L'indice de gras varie fortement en fonction du degré d'engraissement : de 0,8 à 4,3 au tableau XIX. Toutefois la variation parait devenir plus rapide lorsqu'on pousse l'engraissement de façon excessive. En fonction des observations faites, il semble qu'on puisse considérer comme convenables des indices de gras variant entre 1,5 et 2,5 .

TABLEAU $\mathrm{N}^{\circ} \mathrm{XIX}$

\begin{tabular}{|c|c|c|c|c|c|c|c|c|}
\hline Age à 1 'abattage & $\begin{array}{l}19 \\
\text { mois }\end{array}$ & $\begin{array}{l}3 \text { ans } \\
3 \text { mois }\end{array}$ & 4 ans & 4 ans & $\begin{array}{c}5 \text { ans } \\
1 / 2 \\
\text { (n. bas) }\end{array}$ & $\begin{array}{c}5 \text { ans } \\
1 / 2 \\
\text { (n. haut) }\end{array}$ & $\begin{array}{c}7 \text { à } \\
10 \text { ans }\end{array}$ & $\begin{array}{c}10 \text { à } \\
15 \text { ans }\end{array}$ \\
\hline Engraissement & +++ & +++++ & +++++ & ++ & +++ & ++++ & +++ & + \\
\hline $\begin{array}{l}\text { Carcasse } \\
\text { chaude (kg) }\end{array}$ & 174,2 & 209,5 & 223 & 180,6 & 191,4 & 198,1 & 220 & 198,5 \\
\hline $\begin{array}{l}\text { Rendement } \\
\text { commercial }\end{array}$ & 52,8 & 58,8 & 59 & 52,9 & 56,8 & 58,1 & 53,6 & 49,5 \\
\hline Indice de muscle & 19,5 & 20,4 & 19,6 & 19 & 18,9 & 20,6 & 18,7 & 17,7 \\
\hline Indice de gras & 1,48 & 4,3 & 3,8 & 2,2 & 1,77 & 1,82 & 1,48 & 0,8 \\
\hline $\begin{array}{l}\text { Longueur de } \\
\text { carcasse }\end{array}$ & 108,2 & 110,8 & 111,5 & 111 & 115,8 & 113 & 122,4 & 125,5 \\
\hline
\end{tabular}

Il apparaît donc que le lot d'animaux de 5 ans et demi, nourri au niveau "Bas", soit celui qui se rapproche le plus de ce que l'on peut souhaiter aux plans de la qualité des carcasses et de l'économie de l'engraissement

Le lot 7 ans à 10 ans donne aussi des résultats intéressants. C'est donc à la catégorie d'animaux ayant entre 6 et 9 ans, qu'une installation industrielle d'embouche à l'auge, devra de préférence s'adresser.

Les animaux plus jeunes doivent être entretenus trop longtemps et trop engraissés pour atteindre le poids de carcasses requis; les animaux plus âgés sont d'un traitement difficile et onéreux (indice de consommation élevé).

- La qualité de la graisse a été examinée, car l'alimentation intensive à base de maïs, et plus encore à base d'issues de riz fortement huileuses, pouvait avoir une influence (très importante dans l'engraissement du porc).

Le tableau suivant donne les points de fusion et les indices d'iode en indiquant la moyenne, l'erreur type de la moyenne et le nombre d'échantillons.

$$
\mathrm{m} \pm \mathrm{s}_{\mathrm{m} 2}(\mathrm{n})
$$

Ces chiffres confirment l'observation visuelle d'une graisse très ferme, même sous une température élevée, comme à Majunga. 
TABLEAU $\mathrm{N}^{\circ} \mathrm{XX}$

\begin{tabular}{|l|c|c|}
\hline \multirow{2}{*}{ Points de fusion $\left(0^{\circ} \mathrm{C}.\right)$} & $\begin{array}{c}\text { N1 imentation } \\
\text { issues de riz }\end{array}$ & $\begin{array}{c}\text { Alimentation } \\
\text { mais-tourteaus }\end{array}$ \\
\cline { 2 - 3 } & $52,32 \pm 0,084(4)$ & $49,21 \pm 0,383(10)$ \\
\hline Indices d'iode & $29,42 \pm 0,319(4)$ & $29,63 \pm 0,880(10)$ \\
\hline
\end{tabular}

Les résultats obtenus pour l'alimentation aux issues de riz à partir de quatre animaux seulement sont très homogènes. Ils sont corroborés par dix autres examens à partir d'animaux métis nourris de la même manière. (Point de fusion moyen $52,1^{\circ}$.)

Les remaniements d'acides gras dans le rumen sont donc suffisants pour assurer une carcasse d'excellente qualité, même si ceux qui entrent dans l'alimentation appartiennent aux corps gras à bas point de fusion.

\section{APERCU ECONOMIQUE DE L'EMBOUCHE IN'TENSIVE}

Il n'est pas question de faire ci-après une étude économique détaillée mais simplement d'approcher les conditions de rentabilité de cette activité.

Selon nos observations, il faut s'orienter vers l'embouche d'animaux de 5 ans environ pesant presque $300 \mathrm{~kg}$. On a vu qu'en quatre mois on peut espérer leur faire prendre $70 \mathrm{~kg}$ avec une ration d'environ 5,5 U.F. par jour.

Les bovins maigres s'achètent à 30 FMG le $\mathrm{kg}$, les bovins gras se revendent 50 FMG le $\mathrm{kg}$.

- Prix de vente du bceuf gras $360 \times 50$.

18.000 FMG

- Prix d'achat du bouf maigre $290 \times 30$. . . .

8.700 FMG

- Marge . . . . . . 9.300 FMG

Cette marge doit couvrir tous les frais d'achat, frais divers, coût de l'alimentation, coût de la main-d'œuvre, amortissement des installations et assurer un bénéfice.

$\mathrm{Si}$ on considère que les frais dachat et divers se situent aux environs de $1.000 \mathrm{~F}$ par tête, et que le bénéfice doit se situer à environ $1.500 \mathrm{~F}$, le coût maximal de l'opération d'engraissement devient :

$$
9.300-2.500=6.800 \mathrm{FMG}
$$

On considère, dans l'engraissement des bovins, que le coût des aliments représente 75 p. 100 du coût total. Le coût maximal de l'alimentation est alors de 5.100 FMG.

L'animal ayant consommé 660 U.F. pour parvenir au résultat, le coût maximal de l'U.F. est d'environ 7,6 FMG.

Cela permet de voir qu'en fonction des prix pratiqués en 1969 sur le marché malgache des aliments du bétail, pourraient être utilisés :

- Les issues de riz (6 FMG l'U.F.);

- Le manioc sec (6 FMG l'U.F.);

- La mélasse de sucrerie, sous réserve que le transport de la sucrerie au centre d'embouche n'élève pas démesurément le prix rendu au centre;

- Les fourrages verts à haut rendement (6 à 8 FMG 1'U.F.);

- La paille de riz (6 FMG l'U.F.).

Par contre, paraissent trop onéreux :

- Le maîs (12 FMG l'U.F.);

- Le tourteau d'arachide (15 à 20 FMG I'U.F.);

- Le tourteau de coprah (15 FMG l'U.F.);

- Les graines de coton (15 FMG l'U.F.).

Ils ne pourraient être utilisés qu'en faible proportion. L'engraissement des bovins ne nécessite pas une ration très riche en azote; comme nous l'avons vu, l'urée peut valablement en apporter une bonne part à un prix très bas. Il n'est donc pas justifié de consentir aux tourteaux et aux graines légumineuses un prix exceptionnel pour leur richesse azotée, dans le cas qui nous intéresse.

L'ensílage de maïs en épis humides, selon le système Harvestore, aurait une valeur sans doute marginale. Comme cet ensilage n'a jamais été réalisé à Madagascar, nous resterons sur la réserve quant à son coût réel dans ce pays. 
TABLEAU $\mathrm{N}^{\mathrm{D}} \mathrm{XXI}$

Récapiculation des crỗts quotidiens moyens par përiodes de 4 semaines (en g.)

\begin{tabular}{|c|c|c|c|c|c|}
\hline \multirow{2}{*}{2 ans $1 / 2$ Kianjasoa } & 1 & 2 & 3 & 4 \\
\cline { 2 - 6 } & 635 & 727 & 826 & 324 & 650 \\
\hline $3-4$ ans Kianjasoa & 523 & 678 & 853 & 422 & 638 \\
\hline $7-10$ ans Kianjasoa & 535 & 857 & 664 & 585 & 349 \\
\hline 5 ans Miadana (lot Bas) & 419 & 810 & 525 & 478 & 457 \\
\hline 5 ans Miadana (lot Haut) & 661 & 742 & 560 & 432 & 414 \\
\hline $8-16$ mois Miadana & 1.142 & 820 & 975 & 728 & 607 \\
\hline
\end{tabular}

(Sont soulignës les premiers C.Q.M. infërieurs à $500 \mathrm{~g} /$ jour).

Il demeure bien entendu que les problèmes d'approvisionnement sont toujours extrêmement importants, et que des achats massifs sont capables de peser sur les cours d'un marché qui n'est pas très vaste pour certains produits.

Devant ces perspectives, il devient nécessaire d'effectuer des recherches pour essayer d'améliorer la marge bénéficiaire de l'opération.

On s'efforcera de déterminer une technique qui permette de diminuer la durée de l'adaptation des Zébus Malgaches à la claustration et à l'alimentation intensive; on essaiera de composer des rations plus digestes pour abaisser les indices de consommation; on déterminera le gain et la rentabilité obtenus par l'emploi de vitamines, d'oligo-éléments, d'anabolisants, etc.; on s'efforcera de trouver les moyens les plus propices et les plus économiques, capables d'éliminer la tuberculose ou d'en minimiser l'incidence.

\section{LE PROBLEME DES VEAUX}

Il est enfin nécessaire de dire un mot de l'utilisation des veaux. Elle présenterait de nombreux avantages:

1. Les indices de consommation étant faibles, le kilogramme de crôtt est obtenu à meilleur compte;

2. Les animaux abattus avant l'âge de vingt mois fourniraient une viande dont la tendreté serait celle du "baby beef", avec toutes les conséquences que cela comporte au plan de la boucherie;
3. L'incidence de la tuberculose est plus faible sur les animaux jeunes, les enquêtes statistiques l'ont montré;

4. Au plan de l'élevage, les troupeaux seraient plus rapidement débarrassés des mâles, et il est logique de penser que le nombre des femelles pourrait s'accroître d'autant, ce qui augmenterait la productivité d'ensemble du troupeau.

Cependant, il est difficile de prévoir, à l'heure actuelle, quel peut être le devenir de ce projet. Jusqu'ici les veaux n'ont jamais été commercialisés, faute de demande. Ensuite on peut craindre qu'ils ne soient pas capables de marcher longuement comme le font les animaux ayant dépassé deux ans, ce qui impliquerait qu'on doive aller les chercher sur place, avec beaucoup de difficultés de transport.

Pour terminer, disons que l'on ne sait pas si les paysans seraient volontaires pour les vendre en masse, et surtout à quel prix ils seraient vendus.

Il n'est donc pas permis de préjuger de la rentabilité de l'engraissement des veaux, pourtant bien séduisant.

\section{CONCLUSION}

L'engraissement des Zébus Malgaches à l'aide d'une alimentation intensive est possible. A condition de choisir judicieusement les animaux et de viser l'obtention de carcasses de $180 \mathrm{~kg}$ plutôt que de $200 \mathrm{~kg}$ après une em- 
bouche courte (120 jours), en utilisant certains aliments du bétail disponibles sur place à bas prix, il peut sans doute être rentable.

Cela d'autant plus que l'on peut espérer améliorer encore les résultats que nous avons obtenus, en augmentant la qualité et la digestibilité des rations, en mettant au point un protocole d'adaptation des bœufs à l'alimentation intensive plus efficace.

\section{SUMMARY}

Intensive fattening experiments of Malagasy zebu cattle

Malagasy zebu cattle fattening experiments were made, using maïze, peanut cake and rice by-products.

Except very old animals (10 years or more), the adaptability was good.

Daily live weight gain approaches $700 \mathrm{~g}$, during 4 months.

Calves after weaning appear better than olders, but they are not sold by cattle breeders. Five years old animals seem suitable and give good carcasses about $180 \mathrm{~kg}$. On account of the small size of malagasy zebu, it is difficult to obtain $200 \mathrm{~kg}$.

Tuberculosis may be a serious difficulty, With reservation of a judicious choice of animals and diet, fattening may be economically undertaken.

\section{RESUMEN}

\section{Ensayos de engorde intensivo del cebú malgacho}

Se alimentaron de modo intensivo cebues malgachos con raciones conteniendo maiz, torta de cacahuete y salvado de arroz.

Los animales, excepto los bovinos muy viejos (más de 10 años), se adaptan muy bien a ellas.

Se pueden obtener incrementos diarios medios de unos $700 \mathrm{~g}$ por dia durante 4 meses, y despues disminuyen.

Los terneros al destete serian los más ventajosos pero tradicionalmente no se los comercializan.

La utilización de animales de unos 5 años de edad parece más deseable. Permite obtener excelentes canales llegando a $180 \mathrm{~kg}$ facilmente. Es dificil realizar el peso de $200 \mathrm{~kg}$, a causa del tamaño modesto de los cebues malgachos.

La tuberculosis arriesga de constituir un inconveniente formal.

Sin embargo, mediante una elección juiciosa de los animales y de los alimentos, la operación puede ser beneficiosa.

\section{BIBLIOGRAPHIE}

CRAPLET (C.), "Traité d'élevage moderne, t. VIII. La viande de bovins », Paris, Vigot Frères, 1966.

EL SHAFIE (S. A.), * Fattening of Sudan Zebu Cattle. I. Weight gain and carcass analysis of castrated and non castrated Butana calves $\star$, Sudan J. ver. Sci. Anim. Husb., 1965, 6 (1): 33-37.

EL SHAFIE (S. A.), \& Further observation on fattening of Sudan zebu Cattle $»$, Sudan J. vet. Sci. Anim. Husb., 1966, 7 (1): 22-27.

EL SHAFIE (S. A.), McLEROY (G. B.), . Carcass characteristics of feedlot fattened northern and western Sudan Zebu Cattle", Sudan J. vet. Sci. Anim. Husb., 1965, 6 (1) : 3-11.

METZGER (G.), HAMON (J.L.), \& Engraissement précoce et abattage expérimental de trois lots de bovins », Bull. Madagascar, 1959, 9 (157) : 471.

REVIERS (B. de), GILIBERT (J.), SERRES (H.), * Alimentation intensive des zébus à Madagascar. Recherche des potentialités maximales de croissance *, Note de l'I.E.M.V.T., Région de Madagascar, 1967. 


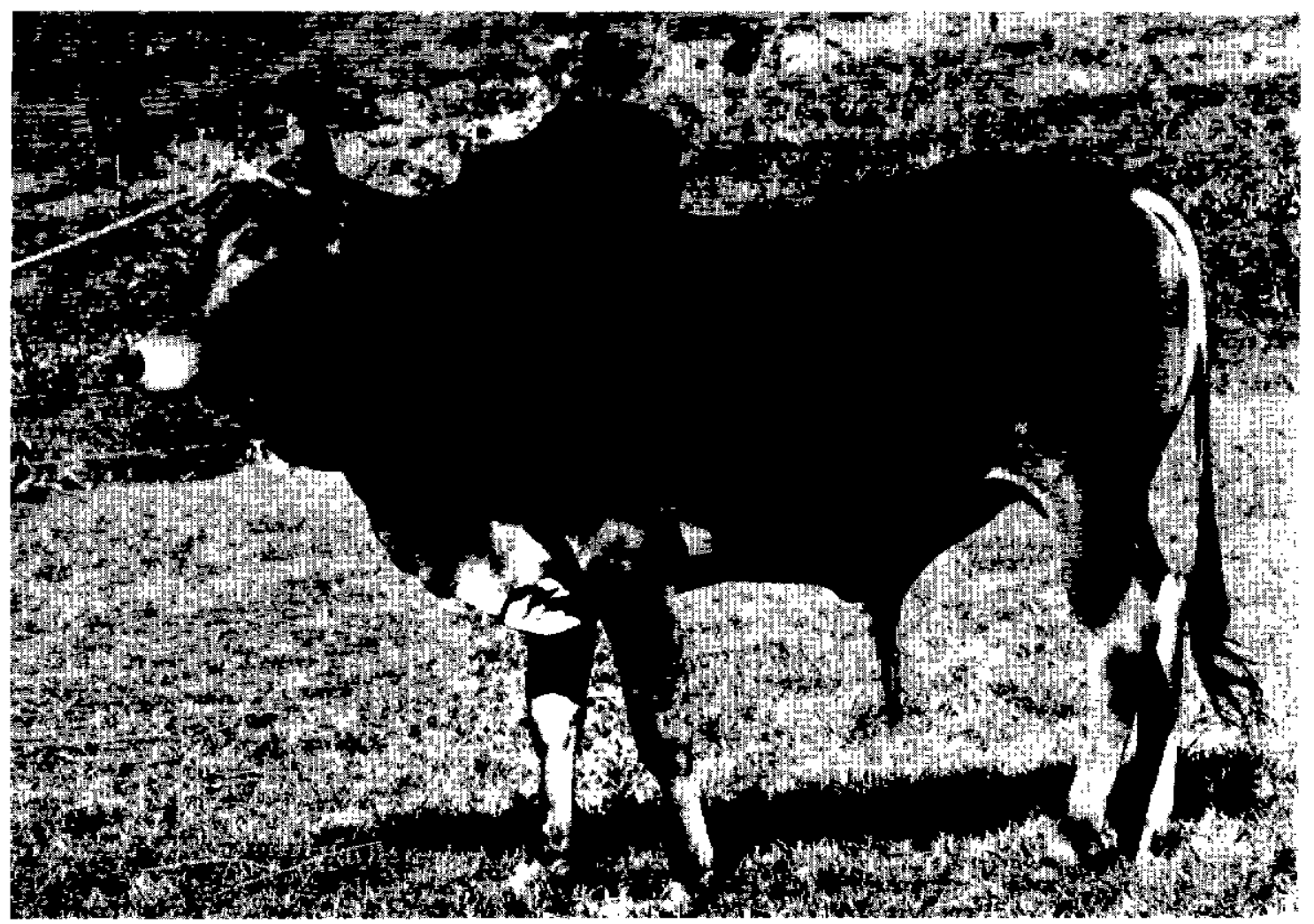

Photo 1. - Bœuf de 3 ans correctement engraissé.

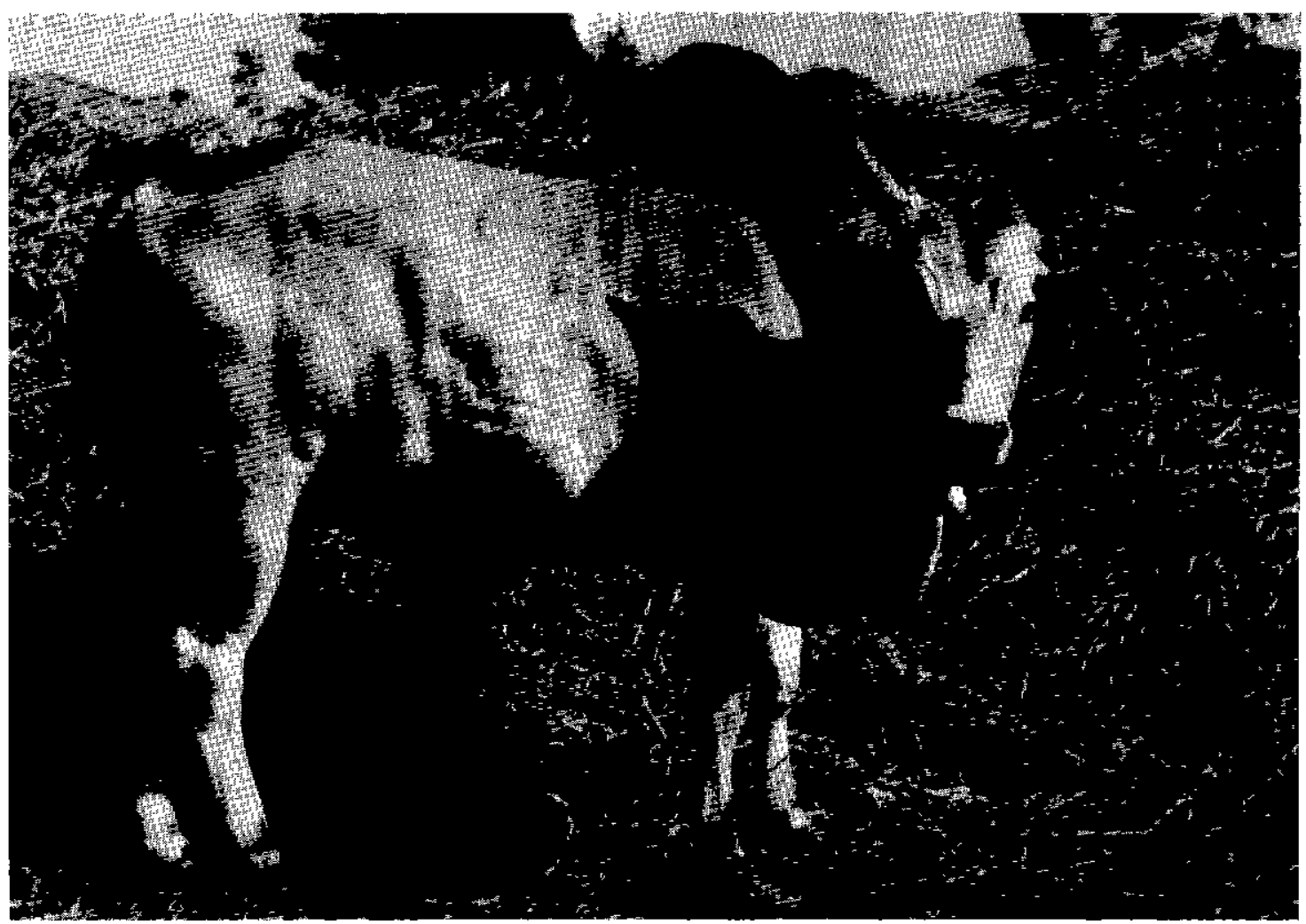

Photo 2. - Bœuf de 3 ans : engraissement excessif. 


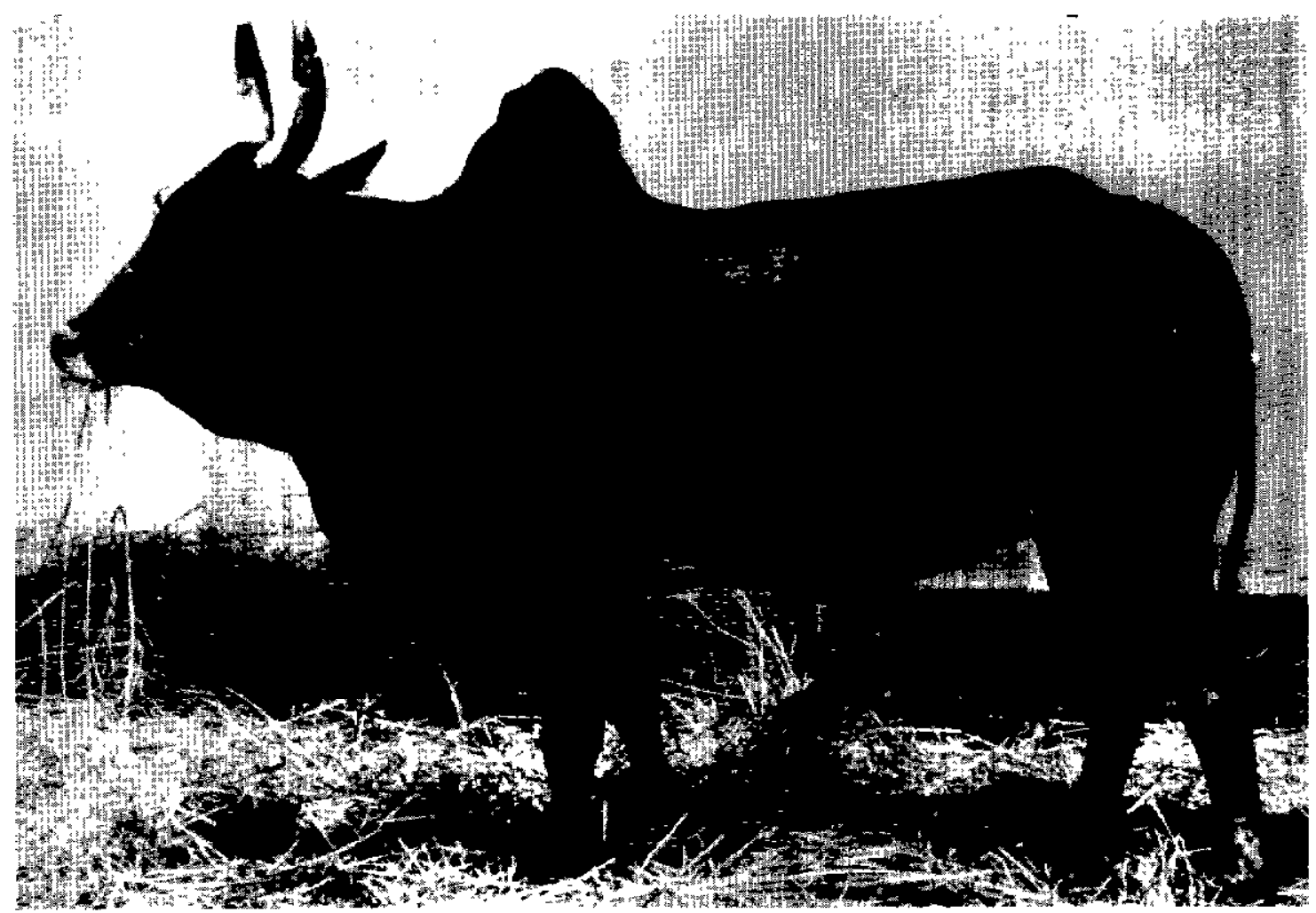

Photo 3. -- Bœuf de 6 ans correctement engraissé.

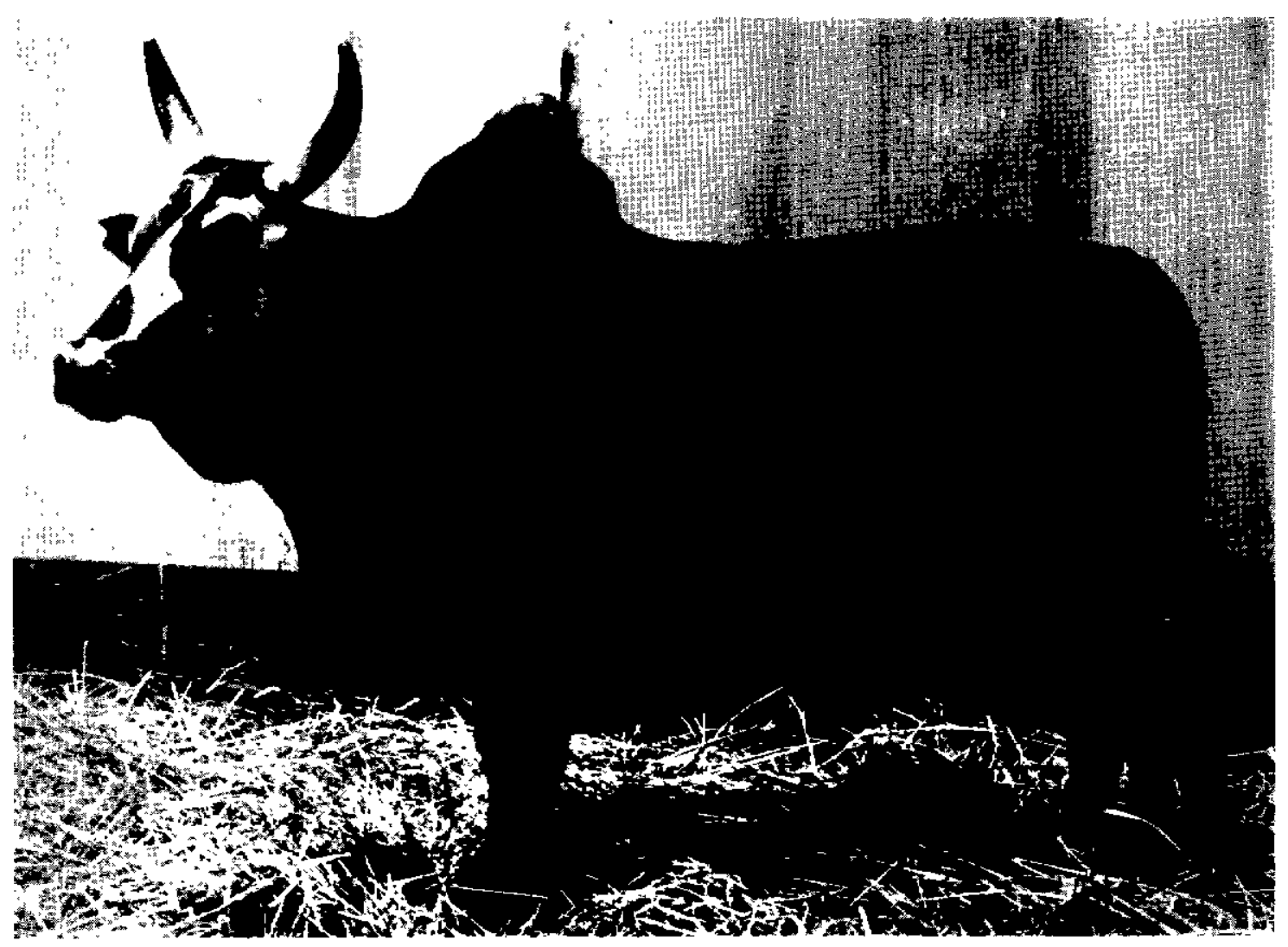

Photo 4. - Bouf de 6 ans : engraissement excessif. 


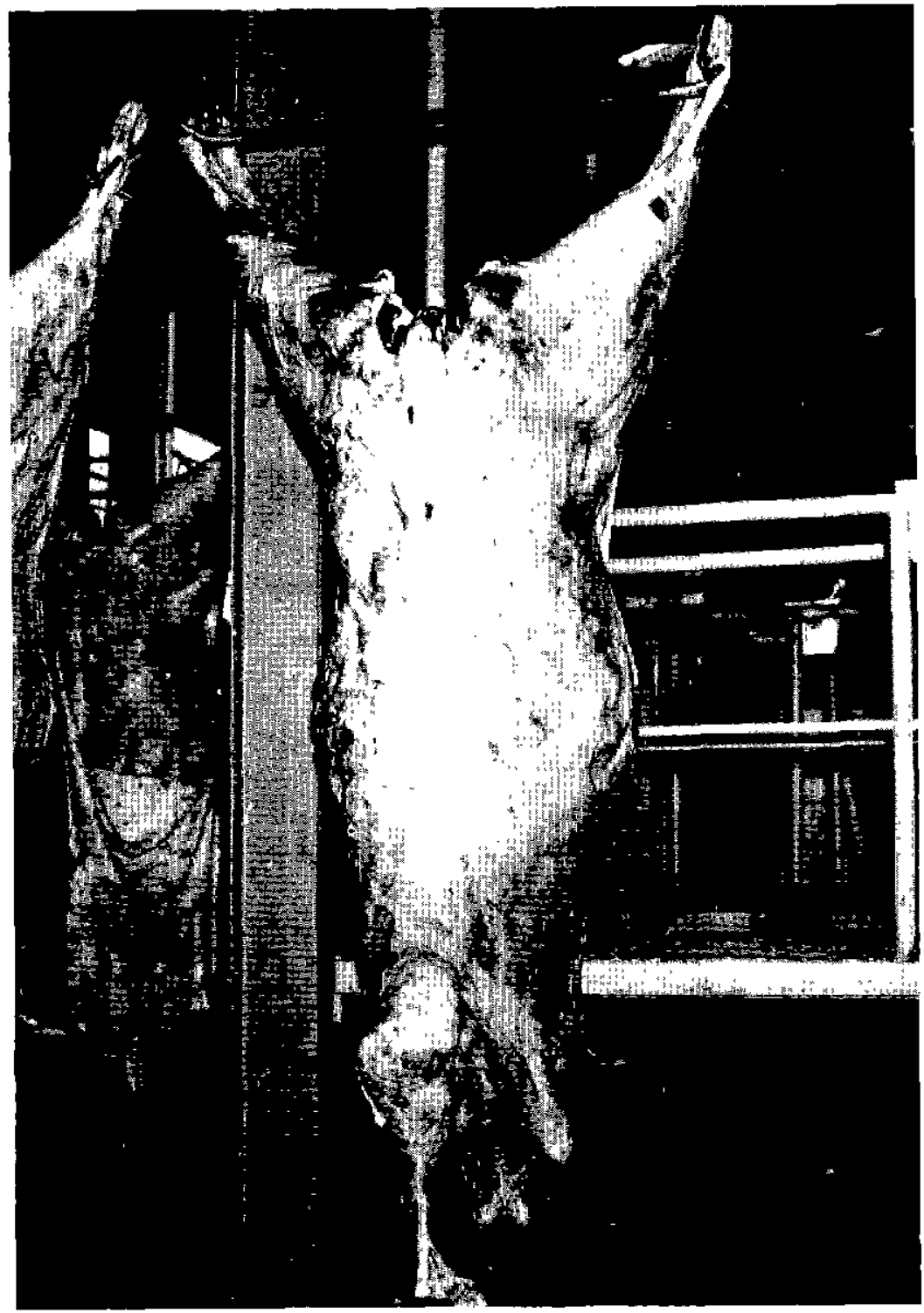

Photo 5. - Type de carcasse à rechercher: couverture homogène mais non excessive, bosse bien développée mais non hypertrophique. 